\title{
Les lasers - Principes fondamentaux
}

\author{
C. Fabre
}

Laboratoire Kastler-Brossel, Université Pierre et Marie Curie, Case 74, 75252 Paris cedex 05, France

Le but de ce cours est de rappeler quelques principes de base de fonctionnement des lasers, et de préciser leurs principales propriétés, qui seront développées par la suite et illustrées sur des exemples, ou mises à profit dans les différentes applications. Il ne prétend évidemment pas à l'exhaustivité, et le lecteur pourra se reporter aux références données en fin de cours pour approfondir les sujets abordés. Nous aborderons vers la fin du cours aussi quelques développements récents de la recherche sur les lasers.

\section{INTRODUCTION}

\subsection{Les ingrédients de base}

Tout système laser comporte nécessairement sous une forme ou sous une autre, les trois éléments de base suivants :

\subsubsection{Un amplificateur optique}

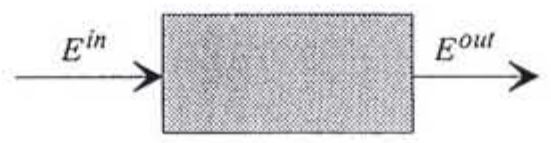

Figure 1

Il s'agit d'un système optique qui lorsqu'il est soumis à un champ oscillant de fréquence $\omega$ $\mathrm{E}^{\text {in }} \cos \omega \mathrm{t}$, fournit à sa sortie un champ $\sqrt{\mathrm{G}} \mathrm{E}^{\text {in }} \cos (\omega \mathrm{t}+\varphi)$ (figure 1), ce que nous écrirons, en utilisant des notations complexes

$$
\mathrm{E}^{\text {out }}=\sqrt{\mathrm{G}} \mathrm{e}^{\mathrm{i \varphi}} \mathrm{E}^{\text {in }}
$$


étant entendu qu'à l'amplitude complexe $\mathrm{E}$ est associée le champ réel $\operatorname{Re}\left(\mathrm{Ee}^{-\mathrm{i} \omega t}\right)$. Un tel dispositif, où il existe une relation de phase bien déterminée entre le champ sortant et le champ entrant est l'analogue dans le domaine optique d'un amplificateur électronique.

\subsubsection{Une boucle de réaction}

Un système optique composé en général de miroirs permet de réinjecter une partie du faisceau de sortie sur l'entrée de l'amplificateur optique. On a donc l'équivalent optique de la boucle de réaction ou de contre-réaction de l'électronique. Ces miroirs constituent une cavité optique, ayant des fréquences de résonance bien déterminées, analogues à une cavité Fabry-Perot. Cette cavité peut être linéaire, (Fig. 2a), ou bien en anneau (Fig. 2b). C'est le couplage entre un système résonnant quantique, constitué par le milieu amplificateur, et un système résonnant électromagnétique, constitué par la cavité, qui est à l'origine des propriétés du laser.

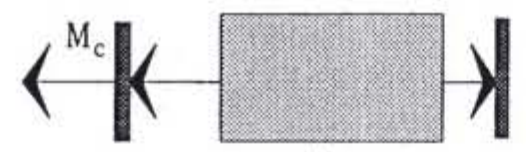

a)

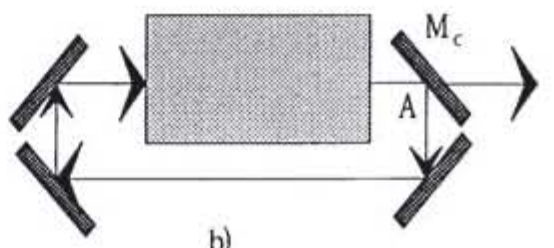

b)

Figure 2

\subsubsection{Un couplage avec l'extérieur}

Dans la plupart des applications on veut disposer d'un faisceau extérieur au laser : il faut donc utiliser un miroir de couplage $\mathrm{M}_{\mathrm{c}}$, de transmission $\mathrm{T} \neq 0$. Le laser est donc intrinsèquement un système ouvert, donc dissipatif. Pour obtenir un régime stationnaire de fonctionnement, il faut fournir à chaque instant dans la cavité l'énergie perdue par le couplage, ce qui est réalisé par apport d'énergie extérieure au niveau de l'amplificateur optique. Le laser est donc à la base un convertisseur d'énergie de pompage en énergie lumineuse. Le rendement de cette conversion est un des paramètres les plus importants d'un laser.

Il faut bien prendre garde que le couplage ne se fait pas à sens unique au niveau du miroir $\mathrm{M}_{\mathrm{c}}$ : le champ laser intracavité est de ce fait sensible aux influences extérieures qui peuvent être transmises à travers $M_{c}$. Il faudra penser dans certains cas à tenir compte de cet effet perturbateur,

\subsection{Conditions d'oscillation}

Considérons dans le modèle simple en anneau de la fig. $2 b$, un rayon circulant à partir du point A situé juste derrière le miroir $\mathrm{M}_{c}$. Si le gain de l'amplificateur est supérieur aux pertes (transmission $T$ du miroir $\mathrm{M}_{\mathrm{c}}$ et pertes en intensité $A$ d'origines variées à l'intérieur même de la cavité), le champ augmente de manière exponentielle à partir de fluctuations initiales du champ électromagnétique toujours présentes à toute fréquence : le laser démarre. L'effet laser est ainsi l'analogue optique de l'effet Larsen bien connu en électroacoustique. 
Dans ce régime de gain linéaire, la puissance intracavité croît sans limite. En fait, le gain de tout système réel n'est pas illimité, ne serait-ce que pour des raisons énergétiques : le gain est donc une fonction décroissante de l'intensité $\left|E^{\text {in }}\right|^{2}$ du champ à l'entrée de l'amplificateur :

$\mathrm{G}=\mathrm{G}\left(\left|\mathrm{E}^{\mathrm{in}}\right|^{2}\right)$.

Le régime stationnaire est atteint lorsque gain et pertes s'équilibrent sur un tour, c'est-à-dire lorsque

$$
G\left(\left|E^{\text {in }}\right|^{2}\right)-1=A+T
$$

Cette condition fixe la valeur de l'amplitude stationnaire du champ intracavité. Le caractère non-linéaire de la réponse du système d'amplificateur à l'onde lumineuse est donc un élément essentiel de fonctionnement du laser en régime stationnaire.

Plusieurs conditions de bouclage sont à considérer pour fixer les valeurs stationnaires de l'oscillation laser :

a- conservation de l'intensité sur un tour : elle nous a donné la condition (1) fixant l'intensité.

b- conservation de la phase de l'oscillation sur un tour : le déphasage de l'onde sur un tour vaut $\omega \frac{\mathrm{L}}{\mathrm{c}}$, où $\mathrm{L}$ est la longueur optique du trajet lumineux sur un tour, et on doit donc avoir :

$$
\omega_{\mathrm{m}} \frac{\mathrm{L}}{\mathrm{c}}=2 \pi \mathrm{m} \text { avec } \mathrm{m} \text { entier }
$$

On obtient, pour les différentes valeurs de $m$, différents modes longitudinaux de l'oscillation laser. La fréquence du laser est une fréquence de résonance de la cavité laser "chaude », c'est-à-dire en présence du champ laser. En effet de manière générale la longueur optique $L$ dépend de l'intensité lumineuse intracavité.

c- conservation de la distribution transverse du champ : l'onde lumineuse intracavité est nécessairement limitée transversalement, et subit dans sa propagation des effets de focalisation ou défocalisation, de diaphragme, et de diffraction. La distribution transverse qui se reproduit identique à elle-même au bout d'un tour de cavité fixe le mode transverse de l'oscillation laser.

Les conditions b et $\mathrm{c}$ sont à l'origine de ce que l'on a coutume d'appeler respectivement la cohérence temporelle et spatiale du champ laser, propriétés sur lesquelles nous reviendrons plus loin. Notons que les conditions a,b,c ne fixent en rien la phase du champ laser qui peut donc prendre n'importe quelle valeur. C'est une caractéristique essentielle de ce dispositif, qui est due au fait que le pompage n'introduit aucune référence de phase a priori dans le système, sur laquelle l'oscillation pourrait se bloquer. Il n'en est pas de même dans d'autres systèmes générateurs d'ondes lumineuses cohérentes, comme par exemple le doublage de fréquence dans une cavité optique. Dans ce dernier cas, il y a aussi amplification lumineuse pour l'onde second harmonique, qui ne peut exister qu'avec une relation de phase bien déterminée avec l'onde de pompe à la fréquence fondamentale.

Après ce rapide survol des caractéristiques de base du laser, il nous faut maintenant rentrer dans le détail de chacun des éléments.

\section{AMPLIFICATEUR OPTIQUE}

Il est basé sur le phénomène d'émission stimulée, ou émission induite, caractéristique essentielle de l'interaction entre un système matériel quantique (atomique, moléculaire, semi- 
conducteur...) et le rayonnement, prédit dès 1916 par Einstein. Nous allons dans cette partie faire une analyse des caractéristiques de base du phénomène d'amplification optique par émission stimulée sur un modèle simple à deux niveaux.

Remarque

Notons que l'émission stimulée n'est pas le seul effet physique conduisant à une amplification de la lumière. L'amplification paramétrique, dans des cristaux non-linéaires, en est un autre. Elle est à l'origine d'un autre type d'oscillateur optique : l'oscillateur paramétrique optique (OPO) dont nous ne ferons pas l'analyse ici. La conjugaison de phase, dans les milieux nonlinéaires comme les vapeurs atomiques, peut conduire aussi à du gain dans l'onde réfléchie, que l'on peut utiliser pour faire fonctionner des oscillateurs à conjugaison de phase.

\subsection{Présentation du modèle simple}

Il s'agit d'un système à 2 niveaux $|a\rangle$ et $|\mathrm{b}\rangle$ ( $|a\rangle$ étant le niveau inférieur), avec pompage et relaxation, schématisé sur la figure (3)

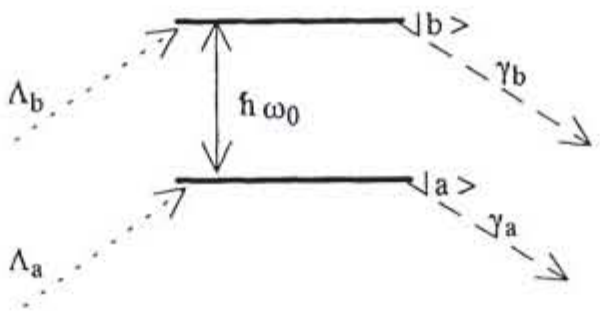

Figure 3

$\omega_{0}$ est la fréquence de Bohr de la transition, $\omega$ la fréquence de l'onde lumineuse (avec $\left.\omega \approx \omega_{0}\right), \gamma_{\mathrm{a}}$ et $\gamma_{\mathrm{b}}$ sont les taux de relaxation des 2 niveaux vers l'extérieur, $\gamma_{\mathrm{ab}}$ le taux de relaxation du dipôle atomique, $\Lambda_{\mathrm{a}}$ et $\Lambda_{\mathrm{b}}$ les taux de pompage des 2 niveaux.

Le système interagit avec le champ électrique de l'onde lumineuse $\mathrm{E}$ cos $\omega \mathrm{t}$ polarisée le long de Ox par une interaction dipolaire électrique correspondant à l'énergie :

$$
\mathrm{V}=-\mathrm{ED}_{\mathrm{x}} \cos \omega \mathrm{t}
$$

$\mathrm{D}_{\mathrm{x}}=\mathrm{qx}$ étant la composante selon $\mathrm{Ox}$ du moment dipolaire du système considéré. Les deux niveaux de la figure 3 font, en fait, partie d'un ensemble plus grand, et ne constituent évidemment pas un système fermé. On ne peut donc pas décrire leur évolution quantique en termes de fonction d'onde $|\Psi\rangle=\mathrm{C}_{\mathrm{a}}|\mathrm{a}\rangle+\mathrm{C}_{\mathrm{b}}|\mathrm{b}\rangle$ et d'équation de Schrödinger, mais à l'aide d'une matrice densité $\sigma$ de dimension 2 :

$$
\sigma=\left[\begin{array}{cc}
\sigma_{\mathrm{aa}} & \sigma_{\mathrm{ab}} \\
\sigma_{\mathrm{ab}} & \sigma_{\mathrm{bb}}
\end{array}\right]
$$

$\sigma_{a a}$ et $\sigma_{b b}$ quantités réelles, représentent les populations des deux états, c'est-à-dire les probabilités d'être dans le niveau $|\mathrm{a}\rangle$ ou $|\mathrm{b}\rangle, \sigma_{2 b}$, nombre complexe appelé cohérence, joue un 
rôle important dans notre problème car elle intervient dans la valeur du dipôle électrique $\left\langle D_{x}\right\rangle$ du système, qui est le médiateur de l'interaction atome-champ électromagnétique :

$$
\left\langle D_{x}\right\rangle=\operatorname{Tr}\left(\sigma \hat{d}_{x}\right)=2 \operatorname{Re}\left(\sigma_{a b} d\right)
$$

où $\mathrm{d}=\left\langle\mathrm{b}\left|\hat{\mathrm{d}}_{\mathrm{x}}\right| \mathrm{a}\right\rangle$ est l'élément de matrice de l'opérateur dipolaire électrique. Dans le cas où il est réel, ce que nous supposons ici, $\left\langle D_{\mathrm{x}}\right\rangle$ est proportionnel à la partie réelle de $\sigma_{\mathrm{ab}}$.

L'évolution de $\sigma$ est la somme de quatre processus distincts :

a-L'évolution libre, sous l'effet de l'hamiltonien purement atomique : les populations sont constantes et la cohérence $\sigma_{\mathrm{ab}}$ est multipliée par un facteur $\mathrm{e}^{\mathrm{i} \omega_{0} \mathrm{t}}$.

b-L'interaction avec le champ électromagnétique, sous l'effet de $\hat{\mathrm{V}}$, qui conduit au phénomène de précession de Rabi : les populations oscillent en particulier au cours du temps avec la fréquence $\mathrm{Ed} / \hbar$ sous l'effet de l'absorption et de l'émission stimulée provoquée par l'onde.

c- L'effet des différents processus de relaxation, qui font tendre les populations et la cohérence vers zéro avec les taux $\gamma_{\mathrm{a}}, \gamma_{\mathrm{b}}, \gamma_{\mathrm{ab}}$.

d- L'effet du pompage extérieur, qui restaure les populations avec des taux $\Lambda_{a}$ et $\Lambda_{b}$.

On peut montrer que les équations d'évolution du système s'écrivent, à l'approximation quasi-résonnante $\left(\omega \approx \omega_{0}\right)$, sous la forme suivante (équations de Bloch) :

$$
\left\{\begin{array}{l}
\frac{d}{d t} \sigma_{b b}=-i \frac{E d}{2 \hbar}\left[e^{i \omega t} \sigma_{b a}-e^{-i \omega t} \sigma_{a b}\right]-\gamma_{b} \sigma_{b b}+\Lambda_{b} \\
\frac{d}{d t} \sigma_{a a}=i \frac{E d}{2 \hbar}\left[e^{i \omega t} \sigma_{b a}-e^{-i \omega t} \sigma_{a b}\right]-\gamma_{a} \sigma_{a a}+\Lambda_{a} \\
\frac{d}{d t} \sigma_{a b}=i \omega_{0} \sigma_{a b}+\frac{i E d}{2 \hbar} e^{i \omega t}\left(\sigma_{b b}-\sigma_{a a}\right)-\gamma_{a b} \sigma_{a b}
\end{array}\right.
$$

\subsection{Réponse stationnaire du système à l’onde laser}

Nous ne nous intéresserons ici qu'au régime stationnaire du système, qu'on détermine en annulant les trois dérivées dans les équations (7). On obtient :

$$
\left\{\begin{array}{c}
\sigma_{\mathrm{bb}}-\sigma_{\mathrm{aa}}=\left(\mathrm{p}_{\mathrm{b}}-\mathrm{p}_{\mathrm{a}}\right)\left[1-\frac{\Omega_{1}^{2}}{\left(\omega-\omega_{0}\right)^{2}+\gamma_{\mathrm{ab}}^{2}+\Omega_{1}^{2}}\right] \\
\sigma_{\mathrm{aa}}+\sigma_{\mathrm{bb}}=\mathrm{p}_{\mathrm{a}}+\mathrm{p}_{\mathrm{b}}+\Omega_{1}^{2} \frac{\gamma_{\mathrm{b}}-\gamma_{\mathrm{a}}}{\gamma_{\mathrm{b}}+\gamma_{\mathrm{b}}\left(\omega-\omega_{0}\right)^{2}+\gamma_{\mathrm{ab}}^{2}+\Omega_{1}^{2}} \\
\sigma_{\mathrm{ab}}=-\mathrm{i} \frac{\mathrm{Ed}}{2 \hbar}\left(\mathrm{p}_{\mathrm{b}}-\mathrm{p}_{\mathrm{a}}\right) \frac{\gamma_{\mathrm{ab}}-\mathrm{i}\left(\omega-\omega_{0}\right)}{\left(\omega-\omega_{0}\right)^{2}+\gamma_{\mathrm{ab}}^{2}+\Omega_{1}^{2}} \mathrm{e}^{\mathrm{i} \omega \mathrm{t}}
\end{array}\right.
$$

Dans ces relations $p_{a}=\frac{\Lambda_{a}}{\gamma_{a}}, p_{b}=\frac{\Lambda_{b}}{\gamma_{b}}$ sont les populations stationnaires des niveaux $|a\rangle$ et lb) en l'absence de champ extérieur, $\Omega_{1}=-\frac{\mathrm{dE}}{\hbar} \sqrt{\frac{\gamma_{\mathrm{ab}}}{\Gamma}}$ est une fréquence caractéristique de l'interaction atome-champ (fréquence de Rabi modifiée), où $\Gamma=\frac{2 \gamma_{\mathrm{a}} \gamma_{\mathrm{b}}}{\gamma_{\mathrm{a}}+\gamma_{\mathrm{b}}}$ est un taux de 
relaxation moyen des populations. On voit sur ces formules que les populations des 2 niveaux prennent les valeurs $p_{a}$ et $p_{b}$ pour $\Omega_{1} \approx 0$, puis sont modifiées par le champ $E$. A très fort champ $\left(\Omega_{1}^{2} \gg \gamma_{\mathrm{ab}}^{2}+\left(\omega-\omega_{0}\right)\right)^{2}$, on observe une égalisation des deux populations $\left(\sigma_{\mathrm{bb}}-\sigma_{\mathrm{aa}} \rightarrow 0\right)$ quel que soit le signe de la différence initiale de population créée par le pompage $p_{b}-p_{a}$, et la cohérence $\sigma_{a b}$ tend vers 0 : c'est la manifestation du phénomène de saturation, qui conduit à une annulation effective à fort champ du couplage entre le champ et le rayonnement. Il y a en effet à ce moment neutralisation entre les effets d'absorption de rayonnement et les effets d'émission induite, qui conduit à une transparence du milieu vis-à-vis de l'onde.

A partir de $\sigma_{\mathrm{ab}}$, on peut calculer à l'aide de (6) le dipôle induit sur l'atome, et, en multipliant par la densité $\mathrm{N}$ de systèmes à 2 niveaux, la polarisation $\mathbf{P}$ du milieu. $\sigma_{\mathrm{ab}}$ étant proportionnel au champ $\mathbf{E}$ appliqué, on peut définir une susceptibilité $\chi$ du milieu par la formule:

$$
\mathbf{P}=\varepsilon_{0} \chi \mathbf{E}
$$

où $\chi=\chi^{\prime}+\mathrm{i} \chi \chi^{\prime \prime}$ est une quantité complexe dont les composantes réelles et imaginaires donnent la réponse en phase et en quadrature du dipôle par rapport au champ appliqué (en notations réelles: $\mathbf{P}=\varepsilon_{0} \mathbf{E}\left(\chi^{\prime} \cos \omega \mathrm{t}+\chi^{\prime \prime} \sin \omega \mathrm{t}\right)$. On a d'après (8):

$$
\begin{aligned}
& \chi^{\prime}=\frac{N d^{2}}{\varepsilon_{0} \hbar}\left(p_{a}-p_{b}\right) \frac{\omega_{0}-\omega}{\left(\omega-\omega_{0}\right)^{2}+\gamma_{a b}^{2}+\Omega_{1}^{2}} \\
& \chi^{\prime \prime}=\frac{N d^{2}}{\varepsilon_{0} \hbar}\left(p_{a}-p_{b}\right) \frac{\gamma_{a b}}{\left(\omega-\omega_{0}\right)^{2}+\gamma_{a b}^{2}+\Omega_{l}^{2}}
\end{aligned}
$$

A faible intensité la susceptibilité complexe vaut :

$$
\chi_{\ell}=\frac{N d^{2}}{\varepsilon_{0} \hbar}\left(\mathrm{p}_{\mathrm{a}}-\mathrm{p}_{\mathrm{b}}\right) \frac{\omega_{0}-\omega+\mathrm{i} \gamma_{\mathrm{ab}}}{\left(\omega-\omega_{0}\right)^{2}+\gamma_{\mathrm{ab}}^{2}}
$$

Elle est proportionnelle à l'inversion de population $p_{a}-p_{b}$ créée par le pompage initial. Lorsque l'intensité augmente, $\chi_{\ell}$ varie à cause des effets de saturation, et l'on a de manière générale la formule très simple suivante

$$
\chi=\chi_{\ell} \frac{\sigma_{\mathrm{aa}}-\sigma_{\mathrm{bb}}}{\mathrm{p}_{\mathrm{a}}-\mathrm{p}_{\mathrm{b}}}
$$

où $\sigma_{a \mathrm{a}}-\sigma_{\mathrm{bb}}$ est la différence de population en présence du champ, telle qu'elle est donnée par la formule (8a).

\subsection{Lien avec les coefficients A,B d'Einstein}

Nous avons utilisé un formalisme de matrice densité, dans lequel la cohérence $\sigma_{a b}$ joue un rôle très important. En particulier la phase relative entre l'oscillation du moment dipolaire électrique, lié à $\sigma_{a b}$, et l'onde incidente est cruciale pour déterminer si le système va absorber de l'énergie ( $\sigma_{b b}-\sigma_{a s}$ augmentant), ou en perdre. Il existe cependant des situations où les équations de Bloch peuvent être transformées en équations portant sur les populations uniquement : c'est le cas où les processus de relaxation sont beaucoup plus efficaces pour les cohérences que pour les populations :

$$
\gamma_{\mathrm{ab}} \gg \gamma_{\mathrm{a}}, \gamma_{\mathrm{b}}
$$


Dans ce cas, les cohérences se trouvent beaucoup plus rapidement en équilibre avec le champ de l'onde incidente que les populations et on peut utiliser à chaque instant leur valeur stationnaire pour déterminer l'évolution des autres variables sur des temps plus longs que $\gamma_{\mathrm{ab}}^{-1}$. on dit qu'on peut éliminer adiabatiquement les cohérences et écrire, en faisant $\mathrm{d}\left(\sigma_{\Delta \mathrm{b}} \mathrm{e}^{-i \omega t}\right) / \mathrm{dt}=0$ dans l'équation (7c) :

$$
\sigma_{\mathrm{ab}}=-\frac{\mathrm{iEd}}{2 \hbar} \frac{1}{\mathrm{i}\left(\omega_{0}-\omega\right)-\gamma_{\mathrm{ab}}}\left(\sigma_{\mathrm{bb}}-\sigma_{\mathrm{aa}}\right) \mathrm{e}^{\mathrm{i} \omega \mathrm{t}}
$$

Les équations pour les seules populations s'écrivent alors :

$$
\left\{\begin{array}{l}
\frac{\mathrm{d}}{\mathrm{dt}} \sigma_{\mathrm{bb}}=-\frac{\mathrm{E}^{2} \mathrm{~d}^{2}}{2 \hbar^{2}}\left(\sigma_{\mathrm{bb}}-\sigma_{\mathrm{aa}}\right) \frac{\gamma_{\mathrm{ab}}}{\left(\omega_{0}-\omega\right)^{2}+\gamma_{\mathrm{ab}}^{2}}-\gamma_{\mathrm{b}} \sigma_{\mathrm{bb}}+\Lambda_{\mathrm{b}} \\
\frac{\mathrm{d}}{\mathrm{dt}} \sigma_{\mathrm{aa}}=\frac{\mathrm{E}^{2} \mathrm{~d}^{2}}{2 \hbar^{2}}\left(\sigma_{\mathrm{bb}}-\sigma_{\mathrm{aa}}\right) \frac{\gamma_{\mathrm{ab}}}{\left(\omega_{0}-\omega\right)^{2}+\gamma_{\mathrm{ab}}^{2}}-\gamma_{\mathrm{b}} \sigma_{\mathrm{aa}}+\Lambda_{\mathrm{a}}
\end{array}\right.
$$

Ces équations couplent seulement population et intensité lumineuse $E^{2}$. On obtient l'évolution en termes de taux de transferts pour les populations $N_{a}=N \sigma_{a a}$ et $N_{b}=N \sigma_{b b}$. Ces équations («rate équations» en anglais) décrivent ainsi un processus de pompage optique sous l'effet du champ appliqué, ne dépendant que de la densité d'énergie électromagnétique $\mathrm{u}=\varepsilon_{0} \mathrm{E}^{2} / 2$. On peut les récrire par exemple pour la population $N_{b}$ sous la forme :

$$
\frac{\mathrm{d}}{\mathrm{dt}} \mathrm{N}_{\mathrm{b}}=\left(\mathrm{B}_{12} \mathrm{~N}_{\mathrm{a}}-\mathrm{B}_{21} \mathrm{~N}_{\mathrm{b}}\right) \mathrm{u}-\mathrm{A}_{21} \mathrm{~N}_{\mathrm{b}}+\mathrm{N} \Lambda_{\mathrm{b}}
$$

Si l'on fait abstraction du terme de pompage, c'est la forme proposée par Einstein pour décrire l'interaction atome-rayonnement. Dans le cas du modèle utilisé ici, on a donc l'expression suivante des coefficients d'Einstein :

$$
B_{12}=B_{21}=\frac{d^{2}}{\varepsilon_{0} \hbar^{2}} \frac{\gamma_{a b}}{\left(\omega_{0}-\omega\right)^{2}+\gamma_{a b}{ }^{2}} \quad A_{21}=\gamma_{b}
$$

On constate ainsi que l'approche par les coefficients d'Einstein du fonctionnement du laser n'est qu'une approximation, valable si le taux d'amortissement de la cohérence $\gamma_{\mathrm{ab}}$ est plus grand que tous les autres taux caractéristiques du problème. C'est le cas de beaucoup de lasers, mais pas de tous.

\subsection{Expression du champ transmis par le milieu}

Le dipôle D créé par l'interaction avec le champ incident est lui-même source d'un nouveau champ électrique rayonné qui s'ajoute au champ incident. Si l'interférence entre ces deux champs dans la direction $\mathrm{Oz}$ de propagation est destructive, il y aura affaiblissement de l'onde, si elle est constructive, il y aura gain, ce que nous recherchons effectivement pour produire l'effet laser. Le champ total dans le milieu est donné par l'équation de Maxwell (projetée sur l'axe $\mathrm{Ox}$ ) en présence d'une polarisation $\mathrm{P}$.

$$
\frac{d^{2}}{d z^{2}} E(z, t)-\frac{1}{c^{2}} \frac{d}{d t^{2}} E(z, t)=\mu_{0} \frac{d^{2} P}{d t^{2}}
$$


Nous faisons ici, pour simplifier, l'approximation d'un milieu infini dans le plan transverse $\mathrm{xOy}$. La solution est donc indépendante de $\mathrm{x}$ et de $\mathrm{y}$, et ne varie que le long de la direction de propagation $\mathrm{Oz}$. Nous négligeons pour simplifier tous les effets transverses, qui peuvent dans certains cas jouer un rôle important.

$P$ étant lié à $E$ par la susceptibilité définie en (9), on obtient l'équation suivante pour l'évolution générale de $E$ :

$$
\frac{d^{2} E}{d z^{2}}-\frac{1}{c^{2}}(1+\chi) \frac{d^{2} E}{d t^{2}}=0
$$

où $\chi$ est lui même une fonction du champ $E$.

\subsubsection{Théorie linéaire}

Plaçons nous tout d'abord en champ faible. $\chi$ est alors indépendant de $\mathrm{E}$ et égal à $\chi_{\ell}$. La solution de l'équation (19) est alors une exponentielle qui s'écrit :

$$
E(z, t)=E_{0} e^{+i(k z-\omega t)}
$$

avec $\mathrm{k}=\frac{\omega}{\mathrm{c}} \sqrt{1+\chi_{\ell}}$. C'est la théorie habituelle de l'indice de réfraction. Si on suppose en outre que le milieu est dilué, la susceptibilité est faible, et on peut écrire :

$$
\mathrm{k} \approx \frac{\omega}{\mathrm{c}}\left(1+\frac{\chi_{\ell}}{2}\right)=\frac{\omega}{\mathrm{c}}\left(1+\frac{\chi_{\ell}}{2}+\mathrm{i} \frac{\chi_{\ell}^{\prime \prime}}{2}\right)
$$

et le champ qui se propage dans le milieu s'écrit finalement :

$$
E(z, t)=E_{0} e^{+i\left(k^{\prime} z-\omega t\right)} e^{-k^{\prime \prime} z}
$$

avec

$$
\mathrm{k}^{\prime}=\frac{\omega}{\mathrm{c}}\left(1+\frac{\chi_{\ell}^{\prime}}{2}\right) \quad \mathrm{k}^{\prime \prime}=\frac{\omega}{\mathrm{c}} \frac{\chi_{\ell}^{\prime \prime}}{2}
$$

Le champ subit donc, lors de sa propagation dans le milieu, un effet de déphasage dépendant de la distance lié à $\chi_{\ell}^{\prime}$ (effet dispersif) et un effet de variation de l'intensité lumineuse lié à $\chi_{\ell}^{\prime \prime}$ :

- Si $\chi_{\ell}^{\prime \prime}$ est positif, il y a absorption. D'après (10), c'est le cas lorsque $p_{a}>p_{b}$.

- Si $\chi_{\ell}^{\prime \prime}$ est négatif, il y a amplification. C'est le cas lorsque $p_{a}<p_{b}$, c'est-à-dire lorsque l'effet du pompage est de créer une inversion de population entre les 2 niveaux de la transition. Remarquons qu'alors $\chi_{\ell}^{\prime}$ change lui aussi de signe : il y a aussi dans ce cas dispersion anormale du milieu.

Calculons la puissance $\mathscr{P}$ cédée en régime permanent par le champ aux atomes par unité de volume.

$$
\mathscr{P}=\overline{\mathrm{E} \frac{\mathrm{dP}}{\mathrm{dt}}}=\varepsilon_{0} \omega \overline{\mathrm{E}^{2} \cos \omega \mathrm{t}\left(-\chi^{\prime} \sin \omega \mathrm{t}+\chi^{\prime \prime} \cos \omega \mathrm{t}\right)}=\frac{1}{2} \varepsilon_{0} \omega \chi^{\prime \prime} \mathrm{E}^{2}
$$

est lié uniquement à la partie imaginaire de la susceptibilité, soit, compte tenu de (11): 


$$
\mathscr{P}=\left(\mathrm{N}_{\mathrm{a}}-\mathrm{N}_{\mathrm{b}}\right) \mathrm{B}_{12} \hbar \omega=\mathrm{B}_{12} \mathrm{~N}_{\mathrm{a}} \hbar \omega-\mathrm{B}_{21} \mathrm{~N}_{\mathrm{b}} \hbar \omega
$$

où l'on a réintroduit les coefficients d'Einstein (16). Cette relation s'interprète très simplement : $\mathscr{P}$ résulte de la compétition de deux effets : l'absorption de quanta d'énergie $\hbar \omega$, avec un taux $N_{a} B_{12}$ et l'émission induite des même quanta, avec un taux $N_{b} B_{21}$.

Le gain en intensité du milieu pour des puissances très faibles du champ incident vaut donc dans ce modèle :

$$
\mathrm{G}_{0}=\mathrm{e}^{-\mathrm{k} x_{i} \mathrm{~L}_{\mathrm{A}}}=\mathrm{e}^{\mathrm{g}_{0} \mathrm{~L}_{\mathrm{A}}}
$$

où $L_{A}$ est la longueur du milieu actif.

\subsubsection{Régime non linéaire - effet de saturation}

En régime d'amplification $\left(\chi_{\ell}^{\prime \prime}<0\right)$, le champ croît en se propageant dans le milieu, et peut finir par atteindre des régimes où $\Omega_{1}^{2} \approx \gamma_{\mathrm{ab}}^{2}+\left(\omega-\omega_{0}\right)^{2}$. On est alors en régime de saturation. L'équation de propagation devient alors beaucoup plus complexe à résoudre, car $\chi^{\prime \prime}$ en particulier dépend de l'intensité $I=|E|^{2}$, et on ne peut plus utiliser le modèle linéaire de la partie (a). On peut mettre cette dépendance sous la forme :

$$
\chi^{\prime \prime}=\chi_{\ell}^{\prime \prime} \frac{1}{1+\mathrm{I} / \mathrm{I}_{\mathrm{s}}} \text { avec } \mathrm{I}_{\mathrm{s}}=\frac{2 \hbar^{2} \gamma_{\mathrm{a}} \gamma_{\mathrm{b}}\left(\gamma_{\mathrm{ab}}^{2}+\left(\omega-\omega_{0}\right)^{2}\right)}{\gamma_{\mathrm{ab}}\left(\gamma_{\mathrm{a}}+\gamma_{\mathrm{b}}\right) \mathrm{d}^{2}}
$$

$I_{s}$ est appelé intensité de saturation du milieu. On montre alors que l'équation de propagation s'écrit, si l'amplitude du champ ne varie pas trop à l'échelle de la longueur d'onde :

$$
\frac{\mathrm{dI}}{\mathrm{dz}}=-\mathrm{k} \chi_{\ell}^{\prime \prime} \frac{\mathrm{I}}{1+\mathrm{I} / \mathrm{I}_{\mathrm{s}}}
$$

Cette équation s'intègre aisément pour donner

$$
\mathrm{Ln} \frac{\mathrm{I}\left(\mathrm{L}_{\mathrm{A}}\right)}{\mathrm{I}(0)}+\frac{\mathrm{I}\left(\mathrm{L}_{\mathrm{A}}\right)-\mathrm{I}(0)}{\mathrm{I}_{\mathrm{s}}}=\mathrm{g}_{0} \mathrm{~L}_{\mathrm{A}}
$$

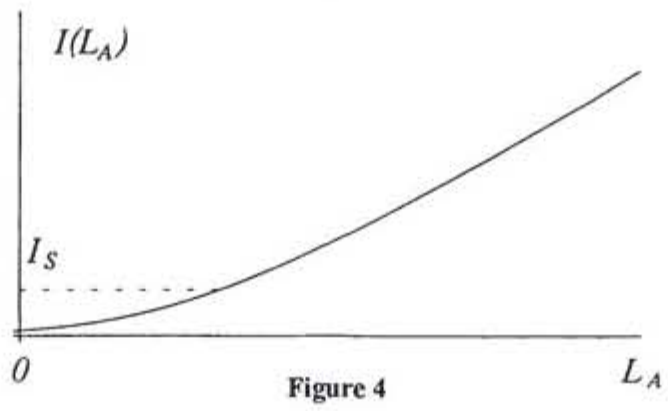

La fonction $I\left(\mathrm{~L}_{\mathrm{A}}\right)$ est donnée sur la figure 4. Lorsque $I\left(L_{A}\right)$ et $I\left(L_{Q}\right)$ sont petits devant $\mathrm{I}_{s}$ on retrouve $I\left(L_{A}\right)=I(0) e^{g_{0} L_{A}}$. Au contraire si $I\left(L_{A}\right) \gg I_{s}$ on a $I\left(L_{A}\right)=I(0)+g_{0} L_{A} I_{s}$. 
L'accroissement du champ est seulement linéaire en fonction de $L_{A}$, au lieu d'être exponentiel. Notons enfin que si $\mathrm{I}(0) \gg \mathrm{I}_{\mathrm{s}}$ on a $\mathrm{I}\left(\mathrm{L}_{\mathrm{A}}\right)=\mathrm{I}(0)\left[1+\mathrm{gL}_{\mathrm{A}}\right]$ avec $\mathrm{g}=\frac{\mathrm{g}_{0}}{1+\mathrm{I} / \mathrm{I}_{\mathrm{s}}}$, tant que $\mathrm{gL}_{\mathrm{A}} \ll<1$ (milieu « mince »).

Remarque :

Nous avons supposé ici qu'il n'y avait qu'une seule onde dans le milieu actif se propageant dans un sens donné : c'est le cas dans les lasers en anneau. Dans le cas de lasers linéaires, le problème est plus complexe, car il se crée une onde stationnaire dans le milieu actif, et par conséquent la saturation au milieu varie fortement d'un point à un autre situé une demilongueur d'onde plus loin («spatial hole burning»). Les différents livres relatifs au laser traitent en détail ce problème.

\subsection{Largeur homogène - Largeur inhomogène}

Dans le modèle simple développé dans cette partie, il est facile de voir d'après les équations (10), que la largeur en fréquence de la bande dans laquelle l'interaction entre le système à deux niveaux et le champ est appréciable et vaut $\Delta \omega_{\mathrm{h}}=\sqrt{\gamma_{\mathrm{ab}}^{2}+\Omega_{1}^{2}}$ autour de la fréquence de Bohr $\omega_{0}$ du système. A basse puissance, ce n'est autre que $\gamma_{a b}$, taux de relaxation de la cohérence entre les niveaux $|a\rangle$ et $|\mathrm{b}\rangle$, dont la valeur minimale est $\frac{\gamma_{a}+\gamma_{b}}{2}$ dans le cas oú la seule cause de relaxation est l'émission spontanée. Dans le régime de saturation, la largeur $\Delta \omega_{\mathrm{h}}$ augmente («élargissement radiatif de la transition ») : à cause de la précession de Rabi induite par le champ entre les niveaux $|\mathrm{a}\rangle$ et $|\mathrm{b}\rangle$, la durée de vie des niveaux est raccourcie. On appelle largeur homogène la largeur $\Delta \omega_{\mathrm{h}}$.

Dans une situation plus réaliste, de nombreux systèmes à deux niveaux participent au processus d'amplification, et n'ont pas tous nécessairement la même fréquence de Bohr $\omega_{0}$. Par exemple, dans le cas des lasers à gaz ( $\left.\mathrm{He}-\mathrm{Ne}, \mathrm{Ar}^{+}, \mathrm{CO}_{2} \ldots\right)$, le milieu actif est formé d'atomes d'ions ou de molécules soumises à l'agitation thermique. A cause du déplacement Doppler de la transition, les différents éléments actifs ont des fréquences de résonance avec l'onde incidente dispersées dans une bande $\Delta \omega_{i}=\frac{\omega_{0}}{\mathrm{c}} \overline{\mathrm{u}}_{\mathrm{th}}=\frac{\omega_{0}}{\mathrm{c}} \sqrt{\frac{\mathrm{k}_{\mathrm{B}} \mathrm{T}}{\mathrm{M}}}$, où $T$ est la température du gaz et $M$ la masse de l'atome, ion ou molécule. Dans le cas de lasers fonctionnant sur des ions en matrice cristalline ( $\mathrm{Nd}$ : YAG, $\mathrm{Ti}$ : Saphir...), les différents ions voient un environnement cristallin différent, qui provoque un déplacement par effet Stark de la fréquence de transition dépendant de la position : on obtient là aussi une dispersion des fréquences de résonance sur un intervalle $\Delta \omega_{\mathrm{i}}$. On appelle largeur inhomogène la largeur $\Delta \omega_{\mathrm{i}}$. Lorsqu'on mesure par exemple l'absorption d'une onde par le milieu (en l'absence de pompage), on obtient une courbe résultant de la superposition de courbes de largeur $\Delta \omega_{h}$ dont les centres sont dispersés sur un intervalle de largeur $\Delta \omega_{\mathrm{i}}$. Nous verrons que le comportement du système présente d'importantes différences selon que $\Delta \omega_{\mathrm{h}}$ ou $\Delta \omega_{\mathrm{i}}$ domine. 


\section{CAVITÉ OPTIQUE}

Le système de contre-réaction par miroir constitue en fait une cavité optique d'un type particulier, dont l'idée a été avancée par Townes et Schawlow dans leur article initiateur sur le laser. Il s'agit d'une cavité Fabry-Perot, dont nous rappelons les principales caractéristiques dans la partie 1. Cette cavité est "ouverte», puisqu'il n'y a pas de parois réfléchissantes parallèles à la direction de propagation du champ. Les caractéristiques transverses du champ maintenu par une telle cavité seront rappelées dans la partie 2.

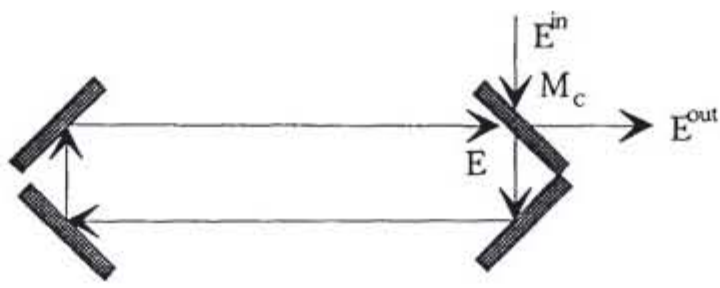

Figure 5

\subsection{Modes longitudinaux}

Supposons donc dans un premier temps que ce sont des ondes planes qui se propagent dans le système de la figure 5 , composé de 3 miroirs parfaits et d'un miroir de couplage $M_{c}$ de coefficients de réflexion et de transmission en intensité $R$ et $T(\operatorname{avec} R+T=1)$.

Soit $E$ l'amplitude complexe du champ intracavité de fréquence $\omega$ juste après le miroir de couplage $\mathrm{M}_{\mathrm{c}}$. Ce champ en se propageant sur le trajet de la cavité est multiplié par un facteur $\mathrm{e}^{\mathrm{i} \varphi} \mathrm{e}^{-\mathrm{A} / 2}$. Dans cette expression, $\varphi$ est le déphasage à la propagation, égal à $\varphi_{0}+\frac{\omega \mathrm{L}}{\mathrm{c}}$, où $\mathrm{L}$ est la longueur optique de la cavité, et $A$ un facteur d'atténuation de l'intensité du champ, traduisant les pertes variées subies par le champ dans la cavité. Juste après le miroir, le champ vaut donc maintenant :

$$
E^{\prime}=\sqrt{T} E^{i n}+\sqrt{R} e^{i \varphi} e^{-A / 2} E
$$

où $E^{\text {in }}$ est le champ injecté dans la cavité à travers $\mathrm{M}_{\mathrm{e}}$. La solution stationnaire pour le champ intracavité s'obtient en égalant $E$ et $E$ ' d'oủ :

$$
E=\frac{\sqrt{T} E^{i n}}{1-\sqrt{R} e^{-A / 2} e^{i \varphi}}
$$

La courbe de variation de $|E|^{2}$ en fonction de $\omega$ pour une valeur fixe de $E^{\text {in }}$ est donnée sur la figure (6)

On obtient des résonances lorsque $\varphi=2 \mathrm{~m} \pi$ avec $m$ entier, c'est-à-dire pour

$$
\omega_{\mathrm{m}}=\frac{\mathrm{c}}{\mathrm{L}}\left(2 \pi \mathrm{m}-\varphi_{0}\right)
$$




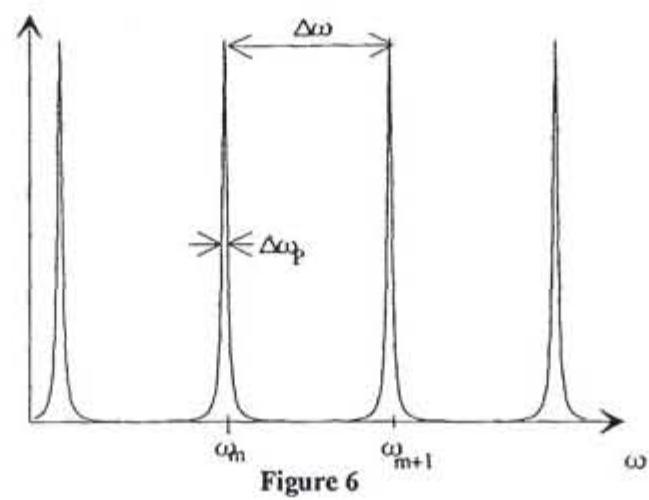

L'intervalle en fréquence $\Delta \omega=\omega_{m+1}-\omega_{m}=2 \pi \frac{c}{L}$ entre 2 résonances consécutives est appelé intervalle spectral libre de la cavité. La largeur des résonances est donnée par

$$
\Delta \omega_{\mathrm{P}}=\frac{2 \mathrm{c}}{\mathrm{L}} \frac{1-\sqrt{\mathrm{R}} \mathrm{e}^{-\mathrm{A} / 2}}{\left(\operatorname{Re}^{-\mathrm{A}}\right)^{1 / 4}}
$$

On a coutume de définir la finesse $\mathrm{F}$, rapport de l'intervalle spectral libre à la largeur des résonances, et qui vaut :

$$
\mathrm{F}=\pi \frac{\left(\operatorname{Re}^{-\mathrm{A}}\right)^{1 / 4}}{1-\sqrt{\operatorname{R}} \mathrm{e}^{-\mathrm{A} / 2}}
$$

Dans ce cas oủ les pertes totales en intensité sur 1 tour, $\mathrm{P}$, dues à la fois à la réflexion sur $\mathrm{M}_{\mathrm{c}}$ et aux pertes intracavité $\left(\mathrm{P}=1-\mathrm{Re}^{-\mathrm{A}} \approx \mathrm{T}+\mathrm{A}\right)$, sont faibles, $\mathrm{F}$ et $\Delta \omega_{\mathrm{p}}$ sont donnés par :

$$
\mathrm{F}=\frac{2 \pi}{\mathrm{P}} \quad \Delta \omega_{\mathrm{P}}=\frac{\mathrm{cP}}{\mathrm{L}}
$$

A résonance exacte, on obtient une amplification du champ intracavité par rapport au champ entrant, caractérisé par une surtension $S$ qui vaut :

$$
\mathrm{S}=\left|\mathrm{E} / \mathrm{E}^{\mathrm{in}}\right|^{2}=\frac{1-\mathrm{R}}{\left(1-\sqrt{\mathrm{R}} \mathrm{e}^{-\mathrm{A} / 2}\right)^{2}} \approx \frac{4 \mathrm{~T}}{\mathrm{P}^{2}}
$$

La dernière expression étant valable dans le cas de faibles pertes totales $P$.

On peut en déduire le champ de sortie du Fabry-Perot, obtenu en écrivant l'équation de couplage sur le miroir de sortie:

$$
E^{\text {out }}=-\sqrt{R} E^{\text {in }}+\sqrt{T E} e^{-A / 2} e^{i \varphi}
$$

Noter le changement de signe du coefficient de réflexion en amplitude par rapport à (30). Ce changement est indispensable pour assurer la conservation de l'énergie entre la somme des champs entrants et la somme des champs sortants sur le miroir $\mathrm{M}_{\mathrm{c}}$. En utilisant (31) et (35) on obtient :

$$
E^{\text {out }}=\frac{e^{-A / 2} e^{i \varphi}-\sqrt{R}}{1-\sqrt{R} e^{-A / 2} e^{i \varphi}} E^{i n}
$$


On obtient pour l'intensité du champ de sortie des résonances en absorption. A la limite où $A=0$, la fraction dans (38) a pour module $1: E^{\text {out }}$ et $E^{\text {in }}$ ont même amplitude pour toute valeur de $\varphi$, ce qui est normal puisque le système n'a pas de pertes dans ce cas.

\subsection{Comportement dynamique de la cavité optique}

Plaçons nous dans le cas de faibles pertes de la cavité $\left(\operatorname{Re}^{-\mathrm{A}} \approx 1-\mathrm{P} \quad \mathrm{P}<<1\right)$. Alors le champ au bout d'un tour vaut, en fonction du champ $E$ :

$$
E^{\prime}=(1-P / 2) e^{i \varphi} E+\sqrt{T} E^{\text {in }}
$$

Plaçons nous à résonance exacte de la cavité $(\varphi=2 \pi \mathrm{m}) . E^{\prime}$ est alors peu différent de $E$. Il est d'autre part égal à $\mathrm{E}\left(\mathrm{t}+\mathrm{T}_{\text {cav }}\right)$ puisque la propagation sur un tour de cavité prend un temps $\mathrm{T}_{\text {cav }}=\frac{\mathrm{L}}{\mathrm{c}}$. On écrira donc :

$$
\mathrm{E}\left(\mathrm{t}+\mathrm{T}_{\text {cav }}\right) \approx \mathrm{E}(\mathrm{t})+\mathrm{T}_{\text {cav }} \frac{\mathrm{dE}}{\mathrm{dt}}=\mathrm{E}(\mathrm{t})(1-\mathrm{P} / 2)+\sqrt{\mathrm{T}} \mathrm{E}^{\text {in }}
$$

Soit :

$$
\mathrm{T}_{\text {cav }} \frac{\mathrm{dE}}{\mathrm{dt}}=(-\mathrm{P} / 2) \mathrm{E}+\sqrt{\mathrm{T}} \mathrm{E}^{\text {in }}
$$

On obtient ainsi l'équation gouvernant la dépendance temporelle du champ intracavité à résonance, lorsque celui-ci varie lentement par rapport à $\mathrm{T}_{\text {cav. }}$. En particulier si $E^{\text {in }}=0$, on obtient la solution transitoire :

$$
E=E(0) e^{-P t / 2 T_{c a v}}
$$

Il s'agit d'une exponentielle amortie, correspondant à l'évacuation progressive de l'énergie dans la cavité. On appellera $\kappa$ le taux d'amortissement de l'amplitude du champ qui vaut donc :

$$
\kappa=\frac{\mathrm{P}}{2 \mathrm{~T}_{\text {cav }}}=\frac{\pi \mathrm{c}}{\mathrm{L}} \mathrm{F}^{-1}=\frac{\Delta \omega_{\mathrm{p}}}{2}
$$

$\kappa^{-1}$ donne l'échelle temporelle caractéristique de l'évolution du champ intracavité.

\subsection{Modes transverses}

Les miroirs étant nécessairement limités transversalement, les modes propres de la cavité Fabry-Perot doivent avoir aussi une extension transversale finie. Or on sait bien, en vertu du principe de Fresnel, qu'une onde d'extension transverse finie diverge lorsqu'elle se propage, à cause de la diffraction. Il faut donc s'opposer à cette divergence par diffraction pour obtenir un mode transverse stable, c'est-à-dire qui se reproduise identiquement à lui-même au bout d'un tour de cavité. On utilise pour cela des éléments optiques focalisants, comme des lentilles, ou l'effet autofocalisateur du milieu amplificateur lui-même. Mais le plus simple est de constituer la cavité avec un ou plusieurs miroirs concaves, qui jouent le rôle de lentilles sans introduire de pertes supplémentaires. Nous allons commencer par étudier ici la configuration la plus simple de cavité avec un seul miroir concave et un miroir plan, puis donner brièvement les résultats de l'étude générale. 


\subsubsection{Modes gaussiens}

Les modes de la cavité sont dans tous les cas déterminés par la résolution de l'équation de Helmholtz :

$$
\Delta \mathrm{E}-\frac{1}{\mathrm{c}^{2}} \frac{\mathrm{E}}{\mathrm{dt}^{2}}=0
$$

avec les conditions aux limites imposées par les miroirs, c'est-à-dire avec un champ $\mathbf{E}$ à l'emplacement des miroirs qui est normal à leur surface.

A l'approximation paraxiale, il existe une solution remarquablement simple à cette équation, qui a la forme suivante, en coordonnées cylindriques $(r, \theta, z)$ autour de l'axe de propagation $\mathrm{Oz}$ :

$$
\begin{gathered}
E(r, z, t)=u(r, z) e^{i(k z-\omega t)} \\
\text { avec } u(r, z)=u_{0}\left(\frac{1}{R(z)}+i \frac{2}{k w^{2}(z)}\right) e^{-r^{2} / w^{2}(z)} e^{i k r^{2} / 2 R(z)} \\
R(z)=z+\frac{z_{R}^{2}}{z} w(z)=w_{0} \sqrt{1+\left(\frac{z}{z_{R}}\right)^{2}} a v e c z_{R}=k w_{0}^{2} / 2
\end{gathered}
$$

Cette solution, qui ne dépend pas de l'angle $\theta$, est présentée sur la figure (7). Elle dépend d'un paramètre $z_{\mathrm{R}}$ qui est appelé longueur de Rayleigh du mode.

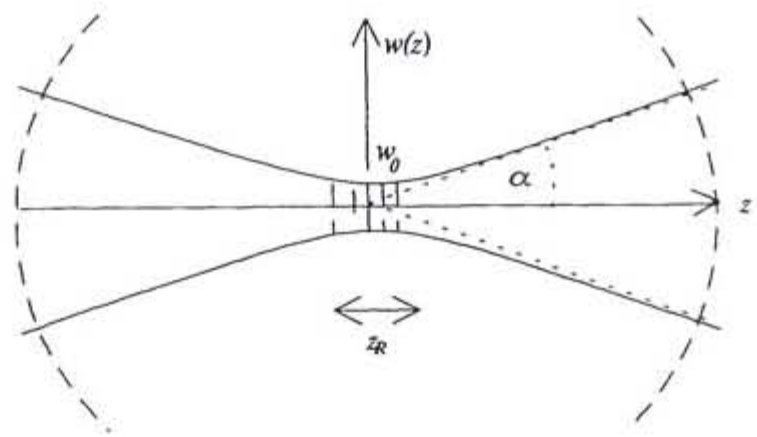

Figure 7

Le mode gaussien est limité transversalement à cause de l'exponentielle $\mathrm{e}^{-\mathrm{r}^{2} / \mathrm{w}^{2}(\mathrm{z})}$. C'est la raison pour laquelle il n'est pas nécessaire d'utiliser des parois réfléchissantes pour confiner latéralement le champ. Le premier facteur donne la variation en phase et en amplitude du champ sur l'axe $\mathrm{Oz}$, Le deuxième facteur donne la variation transverse d'intensité : on a un " faisceau lumineux » dont la dimension transverse $w(z)$ varie comme indiqué par $(45 \mathrm{c})$ avec un minimum égal à $w_{0}$ pour $z=0$ (position de la taille ou «waist » du mode). Comme tout faisceau limité transversalement, le mode gaussien a un comportement divergent pour $|z| \gg z_{R}$. Cette divergence du faisceau se fait dans un cône dont le demi-angle au sommet $\alpha$ vaut $\lambda / \pi w_{0}$. Le dernier facteur donne la variation transverse de phase, qui est la même que celle d'une onde sphérique de rayon de courbure $R(z)$. Au voisinage du waist $\left(|z|<z_{R}\right), R$ est 
très grand et l'onde est pratiquement plane. Loin du waist $|z| \gg z_{R}, R \approx z$, et l'onde tend vers une onde sphérique dont le centre de courbure est fixe en $z=0$. La transition entre les deux régimes se faisant sur la longueur de Rayleigh $z_{\mathrm{R}}$ du mode considéré.

Ce mode gaussien fondamental appelé TEM $\infty_{\infty}$ dépend de deux paramètres $k$ et $w_{0}$. Il n'est pas la seule solution limitée transversalement à l'équation de propagation. Il existe d'autres modes transverses $\mathrm{TEM}_{\mathrm{pq}}$ ayant une dépendance transverse en intensité plus compliquée que l'on trouvera dans les ouvrages spécialisés. Mentionnons ici simplement qu'ils ont aussi un déphasage le long de l'axe, différent du mode $\mathrm{TEM}_{\infty}$. Plus précisément le déphasage entre les champs en deux points différents de l'axe $\mathrm{Oz}, \mathrm{E}(\mathrm{z}, \mathrm{r}=0)$ et $\mathrm{E}\left(\mathrm{z}_{0}, \mathrm{r}=0\right)$, est de la forme $\mathrm{e}^{\mathrm{i} \varphi_{p q}}$ avec :

$$
\varphi_{\mathrm{pq}}=(\mathrm{p}+\mathrm{q}+1) \tan ^{-1}\left(\frac{\mathrm{z}-\mathrm{z}_{0}}{\mathrm{z}_{\mathrm{R}}}\right)
$$

Par contre, tous ces modes ont la même dépendance transverse en phase, donc le même rayon de courbure.

\subsubsection{Cavité plan-concave}

Pour déterminer le mode susceptible d'exister dans une cavité laser, il faut trouver la ou les solutions de l'équation de Helmholtz dont les surfaces d'onde à l'emplacement des miroirs coïncident avec les surfaces de ces miroirs.

Commençons par considérer la cavité la plus simple, constituée d'un miroir plan et d'un miroir concave de rayon de courbure $R_{0}$, distants de $D$. (figure 8 ).

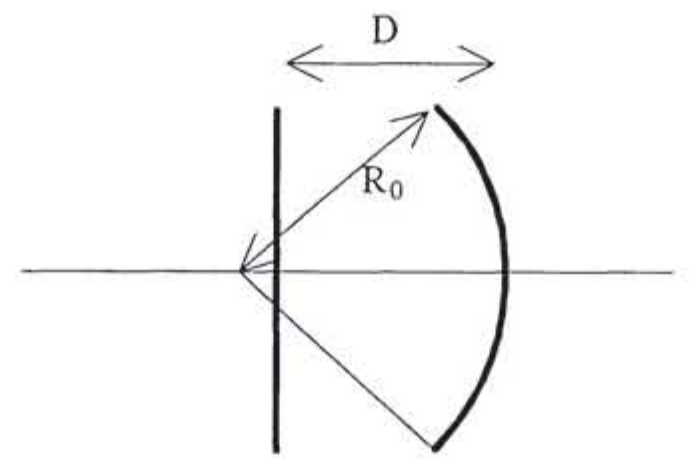

Figure 8

Le mode fondamental de cette cavité est le mode gaussien de la formule (45) oủ le waist est situé sur le miroir plan et tel que $R(z=D)=R_{0}$, soit :

$$
\mathrm{R}_{0}=\mathrm{D}+\frac{\mathrm{z}_{\mathrm{R}}^{2}}{\mathrm{D}}
$$

Cette équation fixe la valeur de la longueur de Rayleigh du mode de la cavité :

$$
\mathrm{z}_{\mathrm{R}}=\sqrt{\mathrm{D}\left(\mathrm{R}_{0}-\mathrm{D}\right)}
$$


qui n'a de solution acceptable que pour $R_{0}>D$. On voit ainsi que toutes les géométries possibles de cavité ne sont pas susceptibles de créer un mode stable. La condition de stabilité remplie, le mode est alors complètement déterminé. Sa fréquence est déterminée par l'équation (32), où $\varphi_{0}$ est le déphasage à la propagation du mode gaussien sur un tour de cavité, calculé à partir de (46), soit :

$$
\omega_{m}=\frac{c}{2 D}\left(2 \pi m-2 \tan ^{-1} \frac{D}{z_{R}}\right)
$$

Sa dimension, et plus précisément celle de son waist, est fixée par :

$$
\mathrm{w}_{0}=\sqrt{\frac{2 \mathrm{c}}{\omega_{\mathrm{m}}}}\left[\mathrm{D}\left(\mathrm{R}_{0}-\mathrm{D}\right)\right]^{1 / 4}
$$

Par exemple un laser avec $D=I m, R_{0}=2 m$ fonctionnant vers $\lambda \approx 1 \mu \mathrm{m}$ a un waist $\mathrm{w}_{0}$ de l'ordre de $0,5 \mathrm{~mm}$. Le miroir plan ayant une transmission non nulle «fait sortir » ce mode avec les caractéristiques transverses données toujours par (45) à l'extérieur de la cavité : on obtient un faisceau dont la divergence $\alpha=\lambda / \pi \mathrm{w}_{0}$ est de $0,5 \mathrm{mrad}$ environ. D'autre part l'intervalle entre 2 modes vaut $150 \mathrm{MHz}$ et la largeur d'une résonance de cavité est de $1 \mathrm{MHz}$ si la transmission de sortie vaut $4 \%$.

La même cavité a pour autres solutions les modes transverses d'ordre supérieur $\mathrm{TEM}_{\mathrm{pq}}$ dont les dimensions sont elles aussi fixées par la géométrie de la cavité. Mentionnons pour finir que le déphasage à la propagation $\varphi_{p \mathrm{q}}$ étant différent pour les différents modes transverses, les fréquences propres $\omega_{\mathrm{pq}}$ de la cavité seront elles aussi différentes pour les différents modes transverses

$$
\omega_{\mathrm{m}, \mathrm{p}, \mathrm{q}}=\frac{\mathrm{c}}{2 \mathrm{D}}\left(2 \pi \mathrm{m}-2(\mathrm{p}+\mathrm{q}+1) \tan ^{-1} \frac{\mathrm{D}}{\mathrm{z}_{\mathrm{R}}}\right)
$$

Il y a donc dégénérescence entre les fréquences propres des différents modes $(m, p, q)$ lorsque $p+q$ est constant, est aussi lorsque $\tan ^{-1} \frac{\mathrm{D}}{\mathrm{z}_{\mathrm{R}}}$ est une fraction rationnelle multipliée par $\pi$.

\subsubsection{Cavité linéaire à deux miroirs}

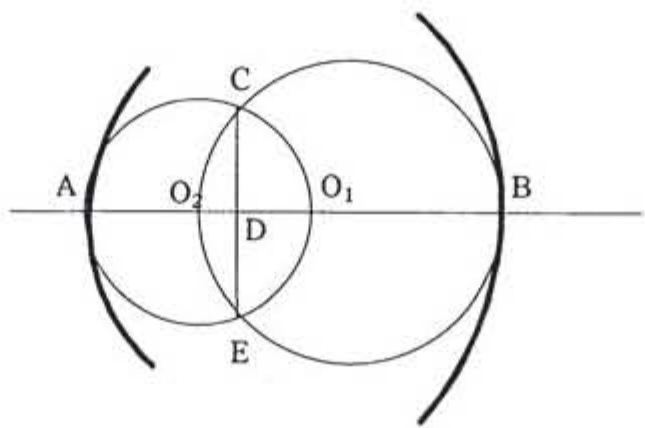

Figure 9 
Pour une cavité linéaire de longueur $D$ fermée par deux miroirs de rayons de courbure $R_{J}$ et $R_{2}$, on peut aussi trouver un mode gaussien de la forme (45) dont la surface d'onde est identique à la surface des miroirs aux deux extrémités de la cavité.

On montre que la condition de stabilité s'écrit alors :

$$
0<\left(1-\frac{\mathrm{D}}{\mathrm{R}_{1}}\right)\left(1-\frac{\mathrm{D}}{\mathrm{R}_{2}}\right)<1
$$

Cette relation a une traduction géométrique simple : il y a stabilité de la cavité lorsque les deux cercles tangents aux deux miroirs en $\mathrm{A}$ et $\mathrm{B}$, et passant par leurs centres de courbure $\mathrm{O}_{1}$ et $\mathrm{O}_{2}$ se coupent. La cote $\mathrm{z}$ de l'intersection de ces deux cercles donne la position du waist du mode. La longueur de Rayleigh vaut :

$$
z_{R}=\frac{\left[D\left(R_{1}-D\right)\left(R_{2}-D\right)\left(R_{1}+R_{2}-D\right)\right]^{1 / 4}}{R_{1}+R_{2}-2 D}
$$

C'est la longueur du segment $\mathrm{CD}$ de la figure (9).

Quelques configurations de cavité jouent un rôle particulier :

- la cavité plan-plan : $R_{1}=\infty \quad R_{2}=\infty$, qui n'est que marginalement stable et pour laquelle la taille du waist n'est pas définie.

- la cavité cofocale : $R_{1}+R_{2}=2 D$ (foyers confondus des 2 miroirs) qui donne le mode dont la taille du waist est maximale (donc la divergence est minimale), à $R_{l}$ et $R_{2}$ fixés.

- la cavité sphérique: $R_{l}+R_{2}=D$ (centres confondus des 2 miroirs), qui n'est que marginalement stable et qui a un waist tendant vers zéro, donc une divergence maximale.

\section{FONCTIONNEMENT DU LASER}

Il nous suffit maintenant de mettre le milieu de la partie B dans la cavité optique de la partie C pour obtenir un laser (fig. 10), dont nous allons étudier plus en détail les caractéristiques

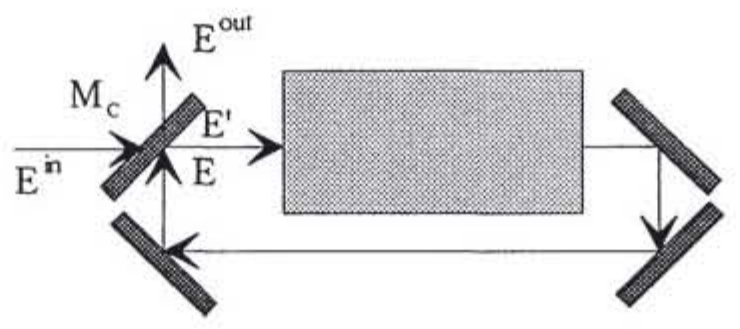

Figure 10

\subsection{Equations du laser}

Nous supposerons ici que le milieu amplificateur ne modifie pas la distribution transverse d'intensité d'un champ qui le traverse. Les propriétés transverses du mode laser sont alors 
uniquement déterminées par la cavité, et on peut appliquer les résultats de la partie 3-3. Nous considérerons alors ici uniquement la valeur du champ E sur l'axe de propagation du mode transverse.

Plaçons nous pour simplifier dans le cas d'un milieu actif mince pour lequel :

$$
E^{\text {out }}=E^{\text {in }} e^{i k \chi^{\prime} L_{A} / 2} e^{g L_{A} / 2}
$$

où $g$ est le gain saturé égal à $-k \chi^{\prime \prime}$. Rappelons que $g, \chi^{\prime}$ et $\chi^{\prime \prime}$ sont des fonctions de l'intensité $\left|\mathrm{E}^{\text {in }}\right|^{2}$. La longueur optique de la cavité en présence de champ vaut donc :

$$
\mathrm{L}=\mathrm{L}_{0}+\frac{1}{2} \chi^{\prime} \mathrm{L}_{\mathrm{A}}
$$

où $L_{o}$ est la longueur de la cavité en dehors du milieu actif. Au bout d'un tour de cavité on a

$$
E^{\prime}=E \sqrt{R} e^{g L_{A} / 2} e^{i \varphi} e^{-A / 2}+\sqrt{T} E^{i n}
$$

où $\mathrm{A}$ est le coefficient d'atténuation en intensité de l'onde dans la cavité, $E$ ' la valeur du champ après propagation sur un temps $T_{\text {cav }}=\frac{L}{c}$ et $\varphi=k L$. Si la variation du champ est faible on peut alors écrire :

$$
\mathrm{E}^{\prime} \approx \mathrm{E}+\mathrm{T}_{\mathrm{cav}} \frac{\mathrm{dE}}{\mathrm{dt}}
$$

On en déduit l'équation donnant la variation de $\mathrm{E}$ dans la cavité

$$
\mathrm{T}_{\text {cav }} \frac{\mathrm{dE}}{\mathrm{dt}}=\left(\sqrt{\mathrm{R}} \mathrm{e}^{\mathrm{i} \varphi+\left(\mathrm{gL}_{\mathrm{A}}-\mathrm{A}\right) / 2}-1\right) \mathrm{E}+\sqrt{\mathrm{T}} \mathrm{E}^{\text {in }}
$$

Remarque : nous avons supposé ici que le gain g à utiliser était celui donné par la solution stationnaire des équations de Bloch. Cela revient à supposer que l'état d'équilibre est atteint beaucoup plus vite pour le milieu matériel que pour la cavité. Ce n'est pas le cas pour tous les lasers. Dans le cas général il faut écrire les équations dynamiques complètes du milieu (équations 7) couplées à l'équation de propagation du champ dans le milieu. La dynamique du milieu est alors gouvernée par les valeurs relatives de 3 taux de relaxation caractéristiques : celui des populations $\gamma_{\mathrm{a}}, \gamma_{\mathrm{b}}$, celui de la cohérence $\gamma_{\mathrm{ab}}$, celui de la cavité $\kappa$ (formule 43). Celle-ci sera étudiée en détail dans le cours de P. Glorieux. La condition de validité de l'équation (58) est :

$$
\kappa<\gamma_{\mathrm{a}}, \gamma_{\mathrm{b}}, \gamma_{\mathrm{ab}}
$$

C'est-à-dire lorsque la largeur de la résonance de cavité est bien inférieure à la largeur de la résonance atomique.

\subsection{Régime stationnaire}

Il est donné par l'annulation de $\frac{\mathrm{dE}}{\mathrm{dt}}$ dans la situation où $E^{\mathrm{in}}=0$, soit :

$$
\left[\sqrt{R} e^{i \varphi+\left(g L_{A}-A\right) / 2}-1\right] E=0
$$


a) On obtient une première solution $E=0$, correspondant au laser éteint. Cette solution n'est pas complètement sans intérêt : le champ laser présente en effet des fluctuations autour de la valeur moyenne nulle qui dépendent du gain du milieu.

b) La solution non nulle pour $E$ correspond à l'annulation du premier facteur, nombre complexe, et donc à deux équations réelles

$$
\left\{\begin{array}{c}
\sqrt{\operatorname{Re}^{(}\left(\mathrm{BL}_{\mathrm{A}}-\mathrm{A}\right) / 2} \cos \varphi-1=0 \\
\sqrt{\mathrm{R}} \sin \varphi=0
\end{array}\right.
$$

système qui a pour solution $\varphi=2 \pi m$ et $\mathrm{Re}^{g \mathrm{LL}_{\mathrm{A}}-\mathrm{A}}=1$. On retrouve bien ainsi les conclusions de l'analyse préliminaire de la partie 1 . La dépendance en intensité du gain est maintenant connue, et l'intensité stationnaire du laser est alors donnée par:

$$
\frac{g_{0} L_{A}}{1-I / I_{s}}=\left(\operatorname{Ln} \frac{1}{R}+A\right) \text { soit } I=I_{s}\left(-1+\frac{g_{0} L_{A}}{\operatorname{Ln}(1 / R)+A}\right)
$$

Cette solution n'est physique $(I>0)$ que pour $\operatorname{Re}^{\mathrm{g}_{0} \mathrm{~L}_{\mathrm{A}}-\mathrm{A}}>1$, c'est-à-dire lorsque le gain au démarrage $\mathrm{e}^{\mathrm{g}_{0} \mathrm{~L}_{\mathrm{A}}}$ est supérieur aux pertes. On trouvera dans le cours de $\mathrm{P}$. Glorieux l'analyse de la stabilité des deux solutions que nous venons de définir.

\subsection{Seuil d'oscillation}

Le seuil du laser correspond au gain minimal pour lequel apparait une solution non nulle de (60) soit :

$$
\left(\mathrm{g}_{0} \mathrm{~L}_{\mathrm{A}}\right)_{\text {seuil }}=\mathrm{Ln}(1 / \mathrm{R})+\mathrm{A} \approx \mathrm{T}+\mathrm{A}
$$

dans le cas où $\mathrm{R} \approx 1$. Si on utilise l'expression du gain linéaire $g_{o}$ obtenu par notre modèle simple de système à deux niveaux (équation 11), on obtient l'expression suivante de l'inversion de population minimale nécessaire à l'oscillation laser :

$$
\left(N_{b}-N_{a}\right)_{\min }=\frac{\omega_{0}^{3}}{3 \pi \Gamma_{s p} c^{2}} \frac{\left(\omega-\omega_{0}\right)^{2}+\gamma_{b a}^{2}}{\omega \gamma_{a b}} \frac{T+A}{L_{A}}
$$

où $\Gamma_{\mathrm{sp}}=\frac{\omega_{0}^{3} \mathrm{~d}^{2}}{3 \pi \varepsilon_{0} \hbar \mathrm{c}^{3}}$ est le taux d'émission spontanée sur la transition $\mathrm{b} \rightarrow \mathrm{a}$. On constate que l'inversion seuil est minimale à résonance exacte. $\mathrm{Si}$ on ne pompe que le niveau supérieur, $\Lambda_{\mathrm{a}}=0$ et le taux de pompage résonnant minimal prend la forme simple suivante :

$$
\left(\Lambda_{\mathrm{b}}\right)_{\min }=\frac{4 \pi}{3} \frac{\gamma_{\mathrm{b}} \gamma_{\mathrm{ab}}}{\Gamma_{\mathrm{sp}}} \frac{\mathrm{T}+\mathrm{A}}{\mathrm{N} \lambda_{0}^{2} \mathrm{~L}_{\mathrm{A}}}
$$

oủ $\lambda_{0}=\frac{2 \pi \mathrm{c}}{\omega_{0}}$ est la longueur d'onde du mode laser résonnant. Il fait intervenir les taux de relaxation de la population de $b$, de la cohérence $\gamma_{\mathrm{ab}}$, ainsi que le nombre d'éléments actifs $\mathrm{N} \lambda_{0}^{2} \mathrm{~L}_{\mathrm{A}}$ présents dans un cylindre de diamètre $\lambda_{0}^{2}$. Cela est dû au fait que la section efficace de diffusion résonnante pour un système à deux niveaux est de l'ordre de $\lambda_{0}^{2}\left(3 \lambda_{0}^{2} / 2 \pi\right.$ plus 
exactement). Notons que $\frac{\gamma_{\mathrm{ab}}}{\Gamma_{\mathrm{sp}}}$ est toujours supérieur à $\frac{1}{2}$ et qu'il est égal à cette valeur lorsque le seul processus de relaxation du système à deux niveaux est l'émission spontanée.

\subsection{Puissance de sortie}

Comme il n'y a pas de champ entrant dans la cavité laser, le champ sortant est simplement $\sqrt{T}$ fois le champ intracavité, soit :

$$
\mathrm{I}^{\text {out }}=\mathrm{TI}_{\mathrm{s}}\left(\frac{\mathrm{g}_{0} \mathrm{~L}_{\mathrm{A}}}{\mathrm{T}+\mathrm{A}}-1\right)=\mathrm{TI}_{\mathrm{s}}\left(\Lambda_{\mathrm{b}} /\left(\Lambda_{\mathrm{b}}\right)_{\text {seuil }}-1\right)
$$

où $\left(\Lambda_{\mathrm{b}}\right)_{\text {seuil }}=\left(\Lambda_{\mathrm{b}}\right)_{\min } \frac{\left(\omega-\omega_{0}\right)^{2}+\gamma_{\mathrm{ab}}{ }^{2}}{\gamma_{\mathrm{ab}}^{2}}$ est le taux de pompage au seuil. Si on s'intéresse à la puissance de sortie (en W/m $/ \mathrm{m}^{2}$ ) elle vaut $\mathrm{P}^{\text {out }}=\frac{1}{2} \varepsilon_{0} \mathrm{E}^{2} \mathrm{c}=\frac{1}{2} \omega_{0} \mathrm{I}$, c'est-à-dire (fig. 11a)

$$
\mathrm{P}^{\text {out }}=\operatorname{TP}_{\mathrm{s}}\left(\Lambda_{\mathrm{b}} /\left(\Lambda_{\mathrm{b}}\right)_{\text {seuil }}-1\right)
$$

avec

$$
P_{\mathrm{s}}=\frac{2 \pi}{3 \lambda_{0}^{2}} \frac{\gamma_{\mathrm{ab}}}{\Gamma_{\mathrm{sp}}} \hbar \omega_{0} \gamma_{\mathrm{b}}
$$
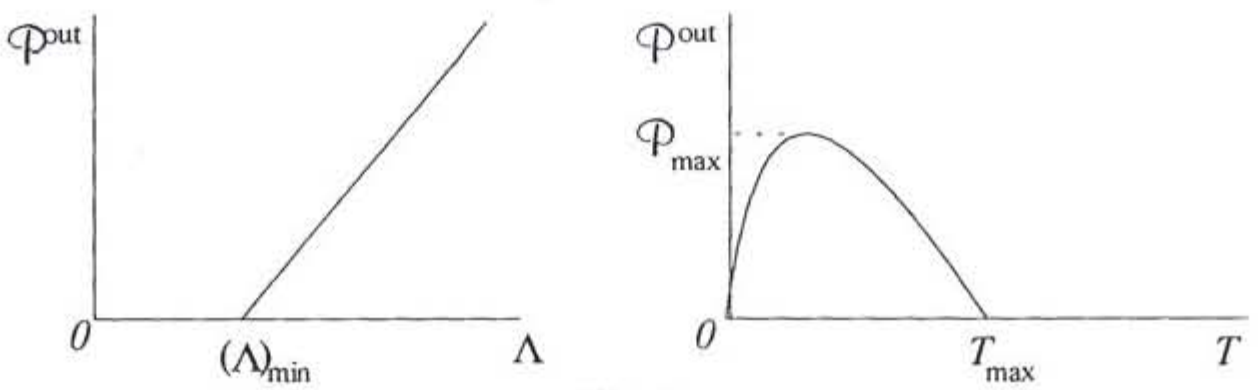

Figure 11

Considérons maintenant la variation de la puissance de sortie en fonction du couplage à l'extérieur, qui n'est autre que la transmission $T$ du miroir de sortie. La figure (11b) donne la variation de $\mathrm{P}^{\text {out }}$ en fonction de $T$. Au delà d'une transmission maximale, les pertes de la cavité sont trop grandes et le laser ne peut osciller. En revanche une valeur de $T$ trop faible couple mal le champ intracavité à l'extérieur. La valeur maximale de la puissance de sortie vaut :

$$
P_{\max }=P_{s} g_{0} L_{A}\left(1-\sqrt{\frac{A}{g_{0} L_{A}}}\right)^{2}
$$

Pour les pertes $A$ intracavité très faibles, elle est égale à la puissance de saturation multipliée par le gain linéaire $g_{0} L_{A}$. 


\subsection{Fréquence de l'onde laser}

Le gain linéaire $\left(g_{0}\right)$ dépendant de la fréquence, la condition de seuil (63) implique qu'on ne peut avoir oscillation que sur une bande de fréquence de largeur finie centrée en $\omega=\omega_{0}$ (fig. 12)

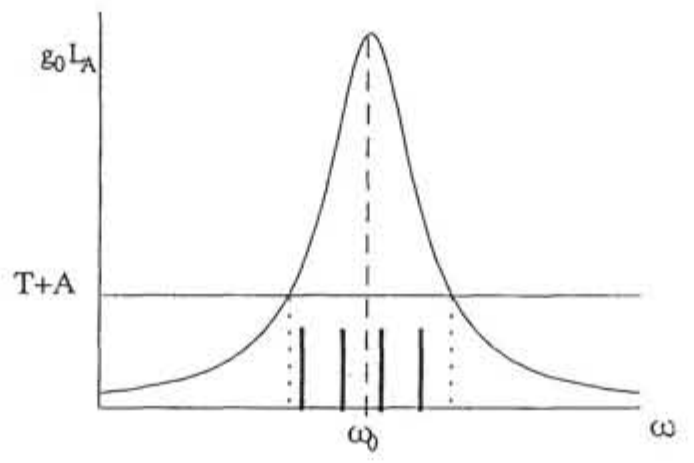

Figure 12

D'autre part, la condition sur la phase $\varphi=2 \pi \mathrm{m}$ impose une condition supplémentaire à la fréquence de l'oscillation. On a en effet, d'après la définition de $\varphi$ :

$$
\varphi=\frac{\omega}{\mathrm{c}} \mathrm{L}=\frac{\omega}{\mathrm{c}}\left(\mathrm{L}_{0}+\frac{1}{2} \chi^{\prime}(\omega) \mathrm{L}_{\mathrm{A}}\right)=2 \pi \mathrm{m}
$$

Cette équation s'écrit encore

$$
\omega=\frac{\omega_{\mathrm{m}}}{1+\frac{\mathrm{L}_{\mathrm{A}} \chi^{\prime}(\omega)}{2 \mathrm{~L}_{0}}} \approx \omega_{\mathrm{n}}\left(1-\frac{\mathrm{L}_{\mathrm{A}}}{2 \mathrm{~L}_{0}} \chi^{\prime}(\omega)\right)
$$

où $\omega_{\mathrm{m}}=\mathrm{m} \frac{2 \pi \mathrm{c}}{\mathrm{L}}$ est la fréquence caractéristique de la cavité vide, telle que nous l'avons étudiée dans le paragraphe 3.2. Nous avons utilisé le fait que le milieu est suffisamment dilué pour que $\chi^{\prime}(\omega)<<1$, ou bien le fait $\mathrm{L}_{\mathrm{A}} / \mathrm{L}_{0} \ll<1$. En utilisant la relation entre $\chi^{\prime}$ et le gain $g$ du milieu, puis la valeur stationnaire du gain $g L_{A}=T+A$ en régime stationnaire du laser, on obtient finalement

$$
\omega=\frac{\omega_{0} \frac{1}{\gamma_{\mathrm{ab}}}+\omega_{\mathrm{m}} \frac{1}{\Delta \omega_{\mathrm{p}}}}{\frac{1}{\gamma_{\mathrm{ab}}}+\frac{1}{\Delta \omega_{\mathrm{p}}}}
$$

où $\Delta \omega_{p}$ est la largeur de la résonance de la cavité vide, donnée par la formule (33). La formule (72) s'interprète simplement en remarquant que $\omega$ est le barycentre des positions des résonances atomiques $\left(\omega_{0}\right)$ et de la cavité $\left(\omega_{\mathrm{m}}\right)$, pondérées par leurs durées de vie respectives. La fréquence de l'oscillation se rapprochera donc plutôt de la plus fine des 2 résonances, c'està-dire, dans la plupart des configurations habituelles, de la résonance de cavité $\omega_{\mathrm{m}}$.

En définitive le laser peut osciller sur un ensemble discret de fréquences, déterminées par l'équation (72) en faisant varier l'entier $m$, dans la bande de fréquence où la condition 
d'oscillation est satisfaite (voir Figure 12). Si on tient compte en plus des modes transverses qui peuvent osciller sur d'autres peignes de fréquence, on voit qu'un laser au dessus du seuil a une tendance naturelle à fonctionner de manière multimode.

\subsection{Fonctionnement en présence d'un champ incident}

Considérons maintenant le cas oủ l'on injecte à l'intérieur de la cavité laser, par le miroir de couplage, un champ $\mathrm{E}^{\text {in }}$ de fréquence $\omega$. On utilise ainsi le système cavité-laser comme un amplificateur du champ incident. Le champ sortant en régime stationnaire se calcule alors à partir du champ entrant $E^{\text {in }}$ et du champ intracavité $\mathrm{E}$ à l'aide de la solution stationnaire de l'équation (68) et de l'équation sur le miroir de couplage :

$$
E^{\text {out }}=-\sqrt{R} E^{\text {in }}+\sqrt{T E} e^{i \phi+\left(g L_{A}-A\right) / 2}
$$

Notez comme en (37) la présence du signe moins devant le coefficient de réflexion $\sqrt{R}$, conséquence de l'égalité des flux incidents et sortants sur le miroir de couplage. On en déduit la valeur du champ de sortie $\mathrm{E}^{\text {out }}$ :

$$
E^{\text {out }}=\frac{e^{i \phi+\left(g L_{A}-A\right) / 2}-\sqrt{R}}{1-e^{i \phi+\left(g L_{A}-A\right) / 2} \sqrt{R}} E^{i n} \approx \frac{i \delta+\left(g L_{A}-A+T\right) / 2}{-i \delta+\left(g L_{A}-A-T\right) / 2} E^{\text {in }}
$$

La dernière expression étant écrite dans le cas particulier simple d'une cavité de grande finesse $(T<<1)$, avec de faibles pertes intérieures $(A<<1)$, d'un milieu amplificateur de faible gain $\left(\mathrm{gL}_{\mathrm{A}}<<1\right)$ et d'un champ injecté quasi résonnant avec la cavité $(\phi=2 \mathrm{~m} \pi+\delta, \delta<<1)$.

\subsection{1. fonctionnement au dessous du seuil (" amplificateur régénératif ")}

Le gain en intensité $\mathrm{G}_{\mathrm{i}}$ de l'amplificateur est donné par le carré du module du coefficient de la formule (74), soit, en supposant le gain du milieu amplificateur purement réel :

$$
\mathrm{G}_{\mathrm{i}}=\frac{\delta^{2}+\left(\mathrm{gL}_{\mathrm{A}}-\mathrm{A}+\mathrm{T}\right)^{2} / 4}{\delta^{2}+\left(\mathrm{gL}_{\mathrm{A}}-\mathrm{A}-\mathrm{T}\right)^{2} / 4}
$$

On voit sur cette expression que, même si le gain $\mathrm{gL}_{\mathrm{A}}$ du milieu actif est faible, $\mathrm{G}_{i}$ peut être aussi grand que l'on veut, à condition d'avoir une valeur de $\mathrm{gL}_{\mathrm{A}}$ proche de $\mathrm{T}+\mathrm{A}$ (" amplificateur régénératif»). Ce que l'on gagne ainsi en gain optique est perdu sur la bande passante de l'amplificateur, qui est alors limitée aux fréquences optiques proches d'une résonance de la cavité laser, alors que l'amplificateur optique sans cavité a une bande passante limitée simplement par la largeur (homogène ou inhomogène) du milieu.

\subsection{2. fonctionnement au dessus du seuil (laser injecté)}

La formule (75) montre que le gain diverge à résonance exacte $(\delta=0)$ lorsque $\mathrm{gL}_{\mathrm{A}}=\mathrm{T}+\mathrm{A}$. On a alors atteint le seuil de l'oscillation laser, oủ le système peut émettre un champ extérieur même en l'absence de champ incident. Mais que se passe-t-il lorsqu'on maintient un champ incident non nul dans ces conditions? Récrivons d'abord les équations du champ intracavité en séparant la phase et l'intensité. On fixera la phase initiale en supposant le champ incident réel $\left(E^{\text {in }}=\sqrt{I^{i n}}\right)$, et on notera $E=e^{i \psi} \sqrt{I}$. On a alors :

$$
\mathrm{e}^{\mathrm{i} \psi} \sqrt{\mathrm{I}}\left\{\left(\mathrm{T}+\mathrm{A}-\mathrm{gL}_{\mathrm{A}}\right) / 2-\mathrm{i} \delta\right\}=\sqrt{\mathrm{TI}^{\mathrm{in}}}
$$


soit encore

$$
\begin{gathered}
\left(-\mathrm{gL}_{\mathrm{A}}+\mathrm{T}+\mathrm{A}\right) \sqrt{\mathrm{I}}=2 \sqrt{\mathrm{TI}^{\text {in }}} \cos \psi \\
\delta \sqrt{\mathrm{I}}=\sqrt{\mathrm{TI}^{\text {in }}} \sin \psi
\end{gathered}
$$

Si le champ incident est nul, l'intensité intracavité $\mathrm{I}_{0}$, comme nous le savons, est fixée par la saturation du gain, et sa phase est une variable libre. Supposons maintenant que le champ incident dans la cavité $\mathrm{TI}^{\text {in }}$ est faible devant le champ intracavité. On traitera alors comme une perturbation au premier ordre l'effet du champ incident sur le point de fonctionnement du laser. On obtient alors les deux équations approchées suivantes pour la phase $\psi$ et l'intensité $\mathrm{I}_{0}+\delta \mathrm{I}$ du champ laser en présence du champ injecté :

$$
\begin{gathered}
\left(-\mathrm{g}\left(\mathrm{I}_{0}+\delta \mathrm{I}\right) \mathrm{L}_{\mathrm{A}}+\mathrm{T}+\mathrm{A}\right) \sqrt{\mathrm{I}_{0}+\delta \mathrm{I}}=2 \sqrt{\mathrm{TI}^{\text {in }}} \cos \psi \\
\sqrt{\mathrm{TI}^{\text {in }}} \sin \psi=\delta \sqrt{\mathrm{I}_{0}+\delta \mathrm{I}} \approx \delta \sqrt{\mathrm{I}_{0}}
\end{gathered}
$$

La deuxième équation donne une valeur déterminée à la phase $\psi$ du champ laser, qui n'existe que si :

$$
\delta^{2}<\mathrm{TI}^{\text {in }} / \mathrm{I}_{0}
$$

On constate donc que si l'onde injectée est suffisamment proche d'une résonance de la cavité laser, ou bien si son intensité est suffisamment grande pour que l'inégalité (79) soit satisfaite, l'émission laser se produit à la fréquence du champ injecté et avec une relation de phase bien déterminée avec celui-ci. Il y a donc un verrouillage du laser de la source extérieure, et le laser " esclave » reproduit alors les fluctuations de phase de la source laser « maître » servant à l'injection. Si l'onde injectée ne satisfait pas au critère (79), le laser n'est pas asservi sur la source extérieure et oscille à la fréquence imposée par la cavité et avec une phase quelconque. Ce procédé d'injection est très souvent utilisé pour conférer à des lasers intenses la cohérence temporelle de lasers monomodes stabilisés de faible puissance utilisés pour l'injection.

\section{DESCRIPTION DES MILIEUX AMPLIFICATEURS}

\subsection{Nécessité d'une inversion de population}

Nous avons présenté dans la partie 2 un exemple simple de milieu susceptible de réaliser l'amplification d'une onde lumineuse. Nous nous proposons ici de généraliser ces résultats à des systèmes plus compliqués, mais plus réalistes, dans lequel les deux niveaux $|a\rangle$ et $\mid$ b $\rangle$ entre lesquels se produit l'oscillation laser font partie d'un ensemble plus grand de niveaux qui participent au processus de pompage.

On peut montrer dans ce cas que l'essentiel des résultats de la partie 2 est conservé, en particulier en ce qui concerne l'absorption et l'émission induite. (Nous reviendrons sur ce point dans le $\S 8.1$ ). De manière générale, une onde de fréquence $\omega$ quasi résonnante avec la transition $|a\rangle \rightarrow|b\rangle$ a une intensité $\mathrm{I}(\mathrm{z})$ qui varie dans le milieu amplificateur selon la loi :

$$
\frac{d I(z)}{d z}=-\alpha I(z)
$$


oủ $\alpha$ est un coefficient réel proportionnel à la différence $N_{a}-N_{b}$ entre les densités d'atomes se trouvant dans le niveau inférieur $a$ et le niveau supérieur $b$ :

$$
\alpha=\left(\mathrm{N}_{\mathbf{2}}-\mathrm{N}_{\mathrm{b}}\right) \sigma(\omega)
$$

$\sigma(\omega)$ quantité positive homogène à une surface, est la section efficace d'absorption du milieu à la fréquence $\omega$ de l'onde. L'intensité varie donc selon la loi :

$$
\mathrm{I}(\mathrm{z})=\mathrm{I}_{0} \exp (-\alpha z)
$$

Il y aura gain dans le milieu lorsque $\alpha<0$, c'est-à-dire pour

$$
\mathrm{N}_{\mathrm{b}}>\mathrm{N}_{2}
$$

Dans cette configuration d'inversion de population l'émission stimulée l'emporte sur l'absorption et l'onde incidente est amplifiée.

Comment réaliser une telle inversion ? On ne peut pas l'obtenir à l'équilibre thermodynamique. En effet dans ce cas $\mathrm{N}_{2}>\mathrm{N}_{b}$ parce que :

$$
\left(\frac{N_{b}}{N_{a}}\right)_{\text {eq.th. }}=\exp -\frac{E_{b}-E_{a}}{k_{B} T}
$$

Il faut donc se placer dans une situation hors d'équilibre, où la cinétique du processus va créer l'inversion. D'une manière générale on doit alimenter (ou pomper) efficacement le niveau $b$ et vider rapidement le niveau a : il n'est en particulier pas possible de pomper directement sur la transition $a \rightarrow b$, car tout processus de pompage agit de manière symétrique sur les transferts $\mathrm{a} \rightarrow \mathrm{b}$ et $\mathrm{b} \rightarrow \mathrm{a}$. Il va avoir pour effet d'égaliser les populations des 2 niveaux $\mathrm{a}$ et $\mathrm{b}$ et ne créera donc pas d'inversion. Il faut donc utiliser une configuration à plus de 2 niveaux. Ceci se réalise de manière plus ou moins simple selon la structure du système quantique considéré. Les paragraphes 5.2 et 5.3 donnent quelques exemples de mécanismes d'inversion de population.

\section{Remarque}

Avant de passer à des systèmes plus compliqués que le système à deux niveaux, remarquons que le modèle simple de la partie 2 peut se rapprocher de situations expérimentales réelles. Il s'agit de dispositifs oú les atomes n'interagissent que transitoirement avec la cavité optique résonnante. C'est en particulier la situation de nombreux masers (à hydrogène, ammoniac, ou atomes de Rydberg) où l'inversion de population est réalisée avant que l'atome ne pénètre dans la cavité et oủ la durée de vie des niveaux $|a\rangle$ et $|b\rangle$ est due par exemple au fait que les atomes ne séjournent qu'un temps fini dans la cavité. Cette situation importante pour la compréhension de l'effet laser lui-même, notamment dans le régime où peu d'atomes et peu de photons participent à l'interaction, n'est pas utilisée dans les configurations laser classiques.

\subsection{Inversion de population dans les systèmes à quatre niveaux}

\subsubsection{Principe}

Dans cette configuration, qui recouvre un grand nombre de lasers usuels, un apport d'énergie extérieure a pour effet de porter le système de son état fondamental $\mathrm{f}$ à un niveau excité e, et cela avec un taux de transition par unité de temps $w\left(N_{\digamma} N_{\odot}\right)$ (il y a en effet ici aussi concurrence entre excitation et désexcitation dues au processus de pompage). 


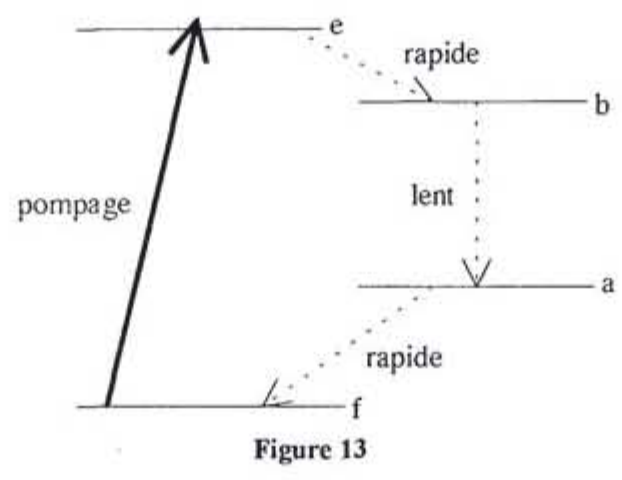

Le système relaxe ensuite rapidement de e vers le niveau supérieur b de la transition laser avec un taux $1 / \tau_{\mathrm{e}}$. Il est ensuite "piégé » dans ce niveau en raison de l'existence d'une relaxation lente $\left(\operatorname{de} \operatorname{taux} 1 / \tau_{b}\right.$ ) du niveau $b$, alors que le niveau a, lui, se vide rapidement avec un taux $1 / \tau_{\mathrm{a}}$ vers le niveau fondamental. On a donc $\tau_{\mathrm{e}}, \tau_{\mathrm{a}} \ll \tau_{\mathrm{b}}$. Il est alors aisé de calculer l'inversion de population au moment du démarrage de l'oscillation laser. En effet dans ce cas les phénomènes d'émission stimulée et d'absorption sur la transition laser ne se produisent pas encore, et les populations des 4 niveaux considérés sont régies par les seuls processus de pompage et de relaxation. Les équations d'évolution de ces populations s'écrivent :

$$
\left\{\begin{array}{c}
\frac{d}{d t} N_{c}=w\left(N_{f}-N_{e}\right)-\frac{N_{e}}{\tau_{e}} \\
\frac{d}{d t} N_{b}=\frac{N_{c}}{\tau_{c}}-\frac{N_{b}}{\tau_{b}} \\
\frac{d}{d t} N_{a}=\frac{N_{b}}{\tau_{b}}-\frac{N_{a}}{\tau_{a}}
\end{array}\right.
$$

L'équation d'évolution de $\mathrm{N}_{\mathrm{f}}$ n'est pas donnée, car il y a aussi conservation de la population totale :

$$
\mathrm{N}_{\mathrm{a}}+\mathrm{N}_{\mathrm{b}}+\mathrm{N}_{\mathrm{c}}+\mathrm{N}_{\mathrm{f}}=\mathrm{N}
$$

La dernière équation du système (85) nous montre qu'en régime permanent on obtient :

$$
\frac{\mathrm{N}_{\mathrm{b}}}{\mathrm{N}_{\mathrm{a}}}=\frac{\tau_{\mathrm{b}}}{\tau_{\mathrm{a}}}
$$

ce qui entraine puisque $\tau_{b} \gg \tau_{2}$ qu'il y a une forte inversion de population quelle que soit l'intensité du pompage $\mathrm{e} \rightarrow \mathrm{f}$. En régime de pompage faible, la fraction des atomes réalisant l'inversion de population vaut :

$$
\frac{N_{b}-N_{a}}{N}=\frac{w \tau_{b}}{1+w \tau_{b}}
$$

Elle est, elle, d'autant plus forte que le pompage est plus efficace. Dans la réalité, il y a toujours possibilité de relaxation des différents niveaux considérés vers d'autres niveaux, qui introduisent des pertes pour le processus de pompage. Si on les inclut dans le modèle, on trouve alors un seuil minimum pour le taux de pompage. Il n'en reste pas moins que le schéma 
de système à 4 niveaux permet de réaliser d'efficaces amplificateurs optiques. Il a souvent été utilisé dans la pratique. Nous passons rapidement en revue dans le paragraphe suivant différents lasers usuels qui fonctionnent sur ce principe.

\subsubsection{Exemples de lasers à 4 niveaux}

\section{* Laser YAG}

Il utilise comme élément actif l'ion $\mathrm{Nd}^{3 *}$ présent sous forme de "dopant " en faible concentration dans un verre ou dans un cristal $\left(\mathrm{Y}_{3} \mathrm{Al}_{5} \mathrm{O}_{12}\right.$, appelé $\left.\mathrm{YAG}\right)$. Le pompage se fait par une source extérieure de lumière, lampe à arc ou diode laser, qui permet d'exciter les ions dans des bandes d'énergie élevées d'où ils sont transférés rapidement, par couplage avec les vibrations du milieu, vers le niveau supérieur de la transition laser. L'émission laser se produit essentiellement sur la transition à $1,06 \mu \mathrm{m}$.

\section{* Lasers Hélium-Néon}

Le laser Hélium-Néon est le premier laser continu à avoir fonctionné. Son mécanisme de pompage implique deux atomes différents (voir figure 14) : une décharge électrique dans le mélange d'hélium et de néon a pour effet essentiel de porter l'atome d'hélium dans des niveaux de longue durée de vie, dits métastables, qui jouent le rôle de réservoir énergétique. Cette énergie est communiquée par collisions aux atomes de néon qui se trouvent portés dans des niveaux excités ayant une énergie voisine de celle des niveaux métastables de l'hélium : il y a inversion de population sur plusieurs transitions, de longueur d'onde $3,39 \mu \mathrm{m}, 1,15 \mu \mathrm{m}$ et $0,633 \mu \mathrm{m}$. L'émission laser est obtenue sur l'une quelconque de ces raies en fonction des miroirs utilisés qui n'ont une bonne réflectivité qu'à une seule de ces longueurs d'onde.

\section{* Lasers accordables}

Les lasers précédents présentent l'inconvénient de fonctionner sur des plages étroites de fréquence, qui ne satisfont pas nécessairement les besoins des utilisateurs. On a donc développé différents types de lasers accordables, qui ont une courbe de gain extrêmement large, dans laquelle on peut sélectionner la fréquence d'oscillation (voir $\S 6.2$ ). Dans de tels milieux lasers, le niveau inférieur de la transition appartient à un contimuum, ou un quasicontimunum de niveaux. Il s'agit par exemple de différentes molécules de colorants en phase liquide ( «lasers à colorants »), ou bien d'ions en matrice cristalline (laser à saphir dopé au Titane, fonctionnant entre $0,7 \mu \mathrm{m}$ et $1,1 \mu \mathrm{m}$ ). Ces différents lasers sont pompés optiquement, en général par un autre laser, qui lui, a une fréquence fixe.

\section{* Lasers moléculaires}

Il existe de nombreux lasers moléculaires émettant dans tous les domaines du spectre depuis l'infrarouge lointain jusqu'à l'ultraviolet. Pour certains de ces lasers la transition a lieu entre différents états vibrationnels de la molécule. Le plus utilisé est le laser à dioxyde de carbone, pompé par décharge électrique et dans lequel les transitions lasers se produisent aux alentours de $10,6 \mu \mathrm{m}$. Dans le laser à fluorure d'hydrogène, émettant vers $3 \mu \mathrm{m}$, l'excitation est de nature chimique : la réaction de formation de la molécule, à partir des molécules d'hydrogène et de fluor, crée directement la molécule dans l'état excité.

La transition laser peut aussi se produire en niveaux électriques moléculaires. Dans ce cas la longueur d'onde émise est souvent dans l'ultraviolet. Par exemple dans les lames à excimères 
comme ArF ou $\mathrm{KrF}$, le niveau inférieur de la transition est dissociatif : il n'existe en effet pas d'état lié pour ces atomes dans le niveau fondamental, alors qu'il y en a dans les niveaux excités : on obtient ainsi directement un dépeuplement extrêmement rapide du niveau inférieur de la transition laser. Ces lasers sont aussi pompés par décharge électrique.

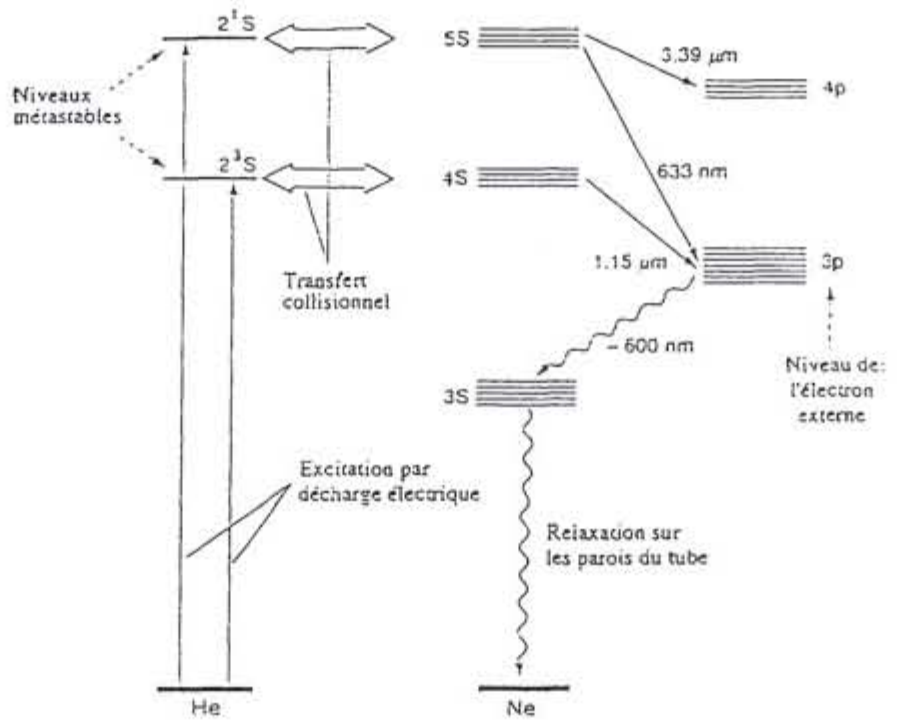

Figure 14

\subsection{Transition laser aboutissant sur le niveau fondamental}

Dans certains lasers, un schéma apparemment plus simple a été utilisé (figure 15), fonctionnant sur seulement trois niveaux. Le pompage est réalisé entre le niveau fondamental a et un niveau excité e. Il est suivi comme dans le cas du laser à 4 niveaux d'une relaxation rapide vers un autre niveau $b$, de longue durée de vie. La différence avec le modèle précédent vient du fait que la transition laser se produit entre ce niveau et le niveau fondamental. Au seuil de l'oscillation laser, les équations cinétiques pour les populations $N_{a}, N_{b}, N_{\epsilon}$ s'écrivent :

$$
\begin{gathered}
\frac{d}{d t} N_{e}=w\left(N_{a}-N_{e}\right)-\frac{N_{e}}{\tau_{e}} \\
\frac{d}{d t} N_{b}=\frac{N_{e}}{\tau_{e}}-\frac{N_{b}}{\tau_{b}} \\
N_{a}+N_{b}+N_{e}=N
\end{gathered}
$$

En régime stationnaire et dans le cas d'un pompage faible $\left(w \tau_{c}<<1\right)$, l'inversion de population est égale à :

$$
\frac{N_{b}-N_{a}}{N}=\frac{w \tau_{b}-1}{w \tau_{b}+1}
$$

résultat à comparer à (88). 


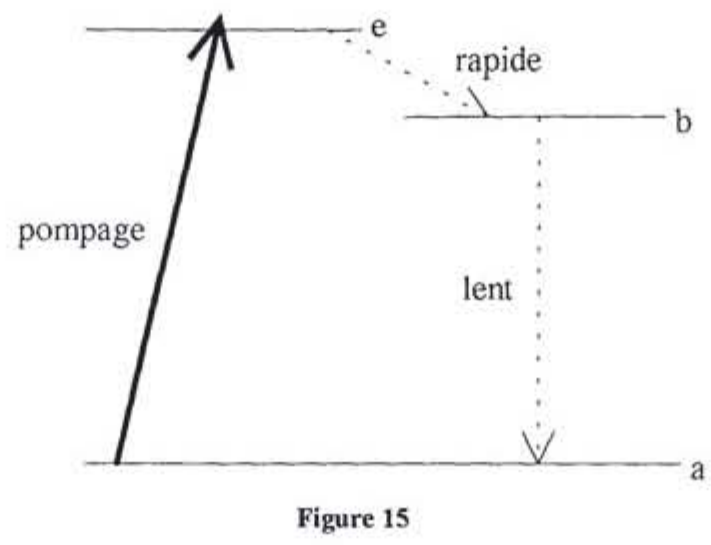

On constate que l'inversion n'est obtenue que pour un pompage suffisamment fort $\left(w \tau_{b} \gg 1\right)$. D'autre part, si on résout les équations dynamiques en présence du champ laser, on constate qu'il est facile d'obtenir un régime $d$ 'oscillations de relaxation : lorsque l'émission laser se produit, l'émission stimulée vide rapidement le niveau b, aboutissant à un arrêt de l'oscillation. Le pompage peut alors de nouveau rétablir au bout d'un certain temps l'inversion de population et le laser redémarre : on obtient donc naturellement un train d'impulsions.

Deux lasers couramment utilisés fonctionnent sur ce schéma : le laser à rubis (ions $\mathrm{Cr}^{3+}$ dans une matrice d'alumine), qui a été le premier laser à fonctionner en 1960, et le laser à Erbium (ion $\mathrm{Er}^{3+}$ dans une matrice de verre de Silice)

Mentionnons pour finir qu'on peut utiliser une autre configuration dans le cas du système à 3 niveaux : celle où la transition a lieu entre les 2 niveaux supérieurs. Cette configuration, très analogue à celle du système à 4 niveaux, est utilisée dans certains lasers fonctionnant dans l'infrarouge lointain.

\section{PROPRIÉTÉS SPECTRALES DES LASERS}

\subsection{Compétition ou coexistence entre modes?}

Nous avons montré dans la partie 4 que la condition d'oscillation (gain non saturé suffisant pour compenser les pertes) pouvait être vérifiée par plusieurs modes longitudinaux du laser. La question est de savoir comment ces différents modes se "partagent » le milieu actif, et en particulier de connaitre les conditions à remplir pour qu'ils puissent coexister, ou bien s'entredétruire. Pour répondre correctement à cette question, il faudrait refaire la théorie complète du milieu amplificateur avec deux champs au lieu d'un. Nous nous contenterons de donner ici quelques idées générales, en remarquant que le mécanisme essentiel est celui de la saturation du gain. En présence de deux champs, on peut montrer que dans de nombreuses situations, le gain pour chacun des modes 1 et 2 peut s'écrire : 


$$
\mathrm{g}_{1}=\frac{\mathrm{g}_{01}}{1+\mathrm{I}_{1} / \mathrm{I}_{\mathrm{S} 11}+\mathrm{I}_{2} / \mathrm{I}_{\mathrm{S} 12}} \quad \mathrm{~g}_{2}=\frac{\mathrm{g}_{02}}{1+\mathrm{I}_{1} / \mathrm{I}_{\mathrm{S} 21}+\mathrm{I}_{2} / \mathrm{I}_{\mathrm{S} 22}}
$$

où on rencontre des termes d'autosaturation, et des termes de saturation croisée. La discussion est plus simple si on considère que dans le régime stationnaire, la saturation n'est pas trop forte. On peut alors développer en série les dénominateurs. Les équations d'évolution des deux modes dans le laser s'écrivent alors de manière générale :

$$
\begin{aligned}
& \frac{\mathrm{dI}_{1}}{\mathrm{dt}}=\left(\alpha_{1}-\beta_{1} \mathrm{I}_{1}-\theta_{12} \mathrm{I}_{2}\right) \mathrm{I}_{1} \\
& \frac{\mathrm{dI}}{\mathrm{dt}}=\left(\alpha_{2}-\beta_{2} \mathrm{I}_{2}-\theta_{21} \mathrm{I}_{1}\right) \mathrm{I}_{2}
\end{aligned}
$$

où $\alpha_{i}(i=1,2)$ est la différence entre gain non-saturé et pertes de la cavité, $\beta_{i}$ le coefficient d'autosaturation et $\theta_{i j}$ les coefficients de saturation croisée pour chacun des deux modes. L'état stationnaire s'obtient en annulant les deux dérivées temporelles dans (92). La solution à ces deux équations est un point dans le premier quadrant du plan $\left(\mathrm{I}_{1}, \mathrm{I}_{2}\right)$ à l'intersection des droites $D_{1}$ d'équation $\left(\alpha_{1}-\beta_{1} I_{1}-\theta_{12} I_{2}=0\right)$ ou $\left(I_{1}=0\right)$, et $D_{2}$, d'équation $\left(\alpha_{2}-\beta_{2} I_{2}-\theta_{21} I_{1}=\right.$ 0 ) ou $\left(I_{2}=0\right)$, marquée par des cercles sur la figure 16. Il faut ensuite analyser la stabilité des solutions ainsi trouvées (voir le cours de P. Glorieux). On trouve alors trois comportements différents (voir figure 16):
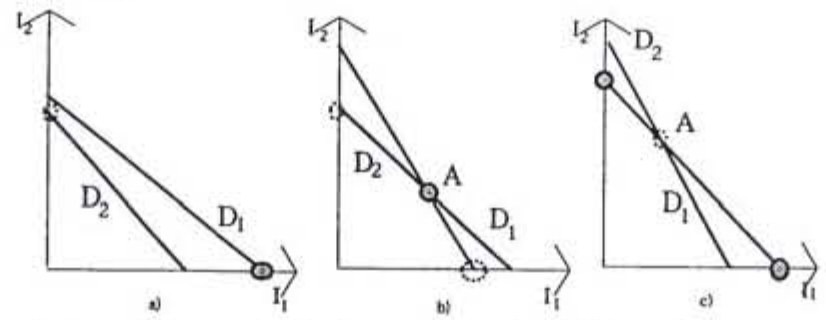

Figure 16. Les solutions stables sont indiquées par un cercle grisé, les solutions instables par un cercle pointillé.

- Si les droites $\left(D_{1}\right)$ et $\left(D_{2}\right)$ ne se coupent pas dans le premier quadrant, la solution stable est sur l'un des deux axes. le laser est donc monomode stable, et le mode qui l'emporte est celui de plus grand gain initial.

- Les droites $\left(D_{1}\right)$ et $\left(D_{2}\right)$ se coupent dans le premier quadrant en un point $A$, mais le couplage entre modes est faible $\left(\left(C=\theta_{12} \theta_{21} / \beta_{1} \beta_{2}<1\right)\right.$. Le point $A$ est un point stable, et le laser est bimode stable.

- Les droites $\left(D_{1}\right)$ et $\left(D_{2}\right)$ se coupent en $A$, mais le couplage entre modes est fort $\left(C=\theta_{12} \theta_{21} / \beta_{1} \beta_{2}>1\right)$. Ce sont les solutions sur les axes qui sont stables, le point $A$ instable. Le laser est monomode bistable, et ce sont les conditions initiales qui déterminent sur quel mode il oscille. Il peut d'autre part commuter de l'un à l'autre sous l'effet d'influences extérieures.

Le comportement du laser est donc régi par la valeur des 6 coefficients de l'équation (92). Ces valeurs dépendent de nombreux paramètres spécifiques de chaque type de laser. par exemple du type d'élargissement de la transition laser. Dans le cas d'un élargissement inhomogène, la saturation de l'amplification n'affecte que les molécules (ou les atomes) du milieu amplificateur dont la fréquence est accordée sur la fréquence lumineuse active : l'effet laser à une fréquence donnée n'affecte que très peu le gain à une autre fréquence. On est en 
régime de couplage faible, et on observera alors l'oscillation simultanée de tous les modes autorisés par la condition de seuil. Cette situation est celle du laser hélium-néon oủ l'élargissement est essentiellement inhomogène (il est dû à l'effet Doppler). Considérons à présent un laser pour lequel la courbe de gain a un élargissement homogène. Dans ce cas, la réponse de chaque atome est affectée de la même façon par la saturation du gain. La courbe de gain va donc être en bloc déplacée vers le bas par suite de la saturation, et la condition d'égalité entre le gain saturé et les pertes n'est réalisée que pour un seul mode. L'oscillation sur ce mode empêche donc l'émission laser sur les autres modes. Le comportement des lasers à gaz tels que $\mathrm{He}-\mathrm{Ne}, \mathrm{Ar}^{+}, \mathrm{CO}_{2}$ basse pression pour lequel l'élargissement est essentiellement inhomogène, est donc très différents de celui des lasers $\mathrm{CO}_{2}$ haute pression, YAG, à colorant, Ti:saphir, semi-conducteurs pour lesquels l'élargissement est homogène.

D'autres phénomènes sont importants pour obtenir un fonctionnement monomode. En particulier, dans la cavité linéaire, l'onde laser est une onde stationnaire, et possède des noeuds et des ventres alternés. Aux ventres l'intensité lumineuse est maximale et le phénomène de saturation diminue le gain. En revanche, l'intensité est nulle aux noeuds et le gain y est donc potentiellement beaucoup plus grand (gain non saturé). La situation est donc tout à fait favorable à l'établissement de l'effet laser pour une deuxième onde stationnaire dont les ventres coïncideraient avec les noeuds de la première. Si le milieu amplificateur est au milieu de la cavité linéaire, il est facile de vérifier que deux modes voisins $\omega_{p}$ et $\omega_{p+1}$ sont précisément dans cette situation, ce qui explique la tendance de tels lasers à osciller sur deux modes. Ce comportement est donc à rapprocher de celui mentionné précédemment dans le cas de la saturation inhomogène du gain mais les trous créés dans la courbe de gain se situent maintenant dans l'espace réel et non plus dans l'espace des fréquences.

\subsection{Fonctionnement monomode longitudinal}

Pour de nombreuses applications, on souhaite avoir une source laser aussi monochromatique que possible. Il faut donc obtenir un laser oscillant sur un seul mode longitudinal (fonctionnement monomode longitudinal). Nous venons de voir qu'un tel régime peut être atteint spontanément dans le cas d'une raie élargie de façon purement homogène, mais cette situation reste exceptionnelle. Une méthode particulièrement simple consiste à utiliser une cavité assez courte pour que l'intervalle entre modes $\mathrm{c} / \mathrm{L}$ soit de l'ordre de la largeur de la courbe de gain : c'est le cas des lasers hélium-néon très courts $(c / L=1 \mathrm{GHz}$ pour $\mathrm{L}=0,3 \mathrm{~m}$ ) ou de certains lasers à semi-conducteurs $(\mathrm{c} / \mathrm{L}=1000 \mathrm{GHz})$. Cette méthode n'est cependant possible que dans des cas favorables.

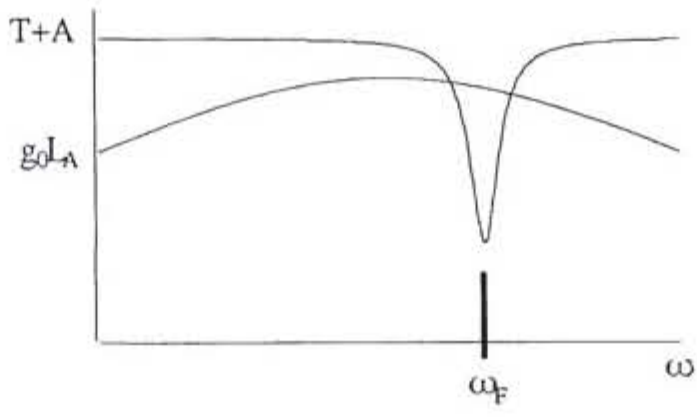

Figure 17 
Le moyen le plus sûr pour rendre un laser monomode consiste à mettre à l'intérieur de la cavité un ou plusieurs éléments sélectifs qui introduisent des pertes dépendant rapidement de la fréquence et ne sont transparents qu'autour d'une fréquence $\omega_{\mathrm{F}}$ (figure 17). La condition d'oscillation n'est donc satisfaite que pour le seul mode longitudinal dont la fréquence est proche de celle de $\omega_{\mathrm{F}}$, que l'on peut choisir en ajustant la position du pic de transmission du ou des filtres.

En fonctionnement monomde, la cohérence temporelle du laser est maximale, et limitée seulement par les fluctuations de longueur optique de la cavité, et de manière ultime, par le phénomène de diffusion de la phase (diffusion Schawlow-Townes), qui sera décrit en détail dans le cours sur les fluctuations quantiques dans les lasers

\section{LASERS EN IMPULSION}

Dans ce paragraphe nous exposons différentes méthodes permettant de produire des impulsions courtes dans les systèmes lasers. Celles-ci permettent d'atteindre, pour une puissance de pompage donnée, des puissances instantanées de l'onde laser très grandes au moment du sommet de l'impulsion et donc de très intenses champs électriques.

\subsection{Laser à modes synchronisés}

Considérons un laser continu émettant sur un grand nombre $N$ de modes de fréquences $\omega_{\mathrm{k}}=\omega_{0}+\mathrm{k} 2 \pi \mathrm{c} / \mathrm{L}(k=0, \ldots \ldots, N-I)$. Le champ électrique émis par un tel laser peut donc s'écrire :

$$
E(t)=\sum_{k=0}^{N-1} a_{0} \cos \left(\omega_{k} t-\phi_{k}\right)
$$

et son intensité est donnée par :

$$
I(t)=\frac{N a_{0}^{2}}{2}+a_{0}^{2} \sum_{j>k} \cos \left[\left(\omega_{j}-\omega_{k}\right) t+\phi_{k}-\phi_{j}\right]
$$

où $\varphi_{\mathrm{k}}$ sont les phases des différents modes. En général, comme nous avons vu dans le paragraphe 6.1 , les modes ne peuvent coexister que s'ils s'ignorent mutuellement, et en conséquence ces phases sont des variables aléatoires incorrélées. Dans ce cas le deuxième terme de (94) est nul en moyenne, et l'intensité moyenne vaut $\overline{\mathrm{I}}=\mathrm{Na}_{0}^{2} / 2$. Le deuxième terme est en revanche responsable de fluctuations autour de cette valeur, dont l'écart quadratique moyen vaut $\Delta \mathrm{I}=\overline{\mathrm{I}}$. Un tel laser multimode présente donc un "bruit » d'intensité important autour de sa valeur moyenne.

Il est cependant possible de corréler entre eux les différents modes. Une méthode souvent utilisée consiste à insérer dans la cavité laser un modulateur qui introduit des pertes oscillant à la fréquence $\Delta=2 \pi \mathrm{c} / \mathrm{L}$ égale à l'intervalle entre modes. La modulation crée pour chacun des modes de fréquence $\omega_{k}$ des bandes latérales de fréquence $\omega_{k} \pm \Delta=\omega_{k \pm}$ c'est-à-dire identique aux fréquences des modes voisins. Un phénomène d'injection, analogue à celui décrit au paragraphe 4.6.2 se produit dans certaines conditions, qui aboutit à une mise en phase de ces différents modes. Supposons que toutes les phases $\varphi_{k}$ prennent une valeur commune $\varphi_{k}=\varphi$. Les différents modes interfèrent alors soit constructivement soit destructivement en fonction du temps. Un calcul simple permet d'obtenir l'expression suivante pour l'intensité (figure 18) : 


$$
I(t)=\frac{a_{0}^{2}}{2}\left(\frac{\sin \left(N \Delta \frac{t}{2}\right)}{\sin \left(\Delta \frac{t}{2}\right)}\right)^{2}
$$

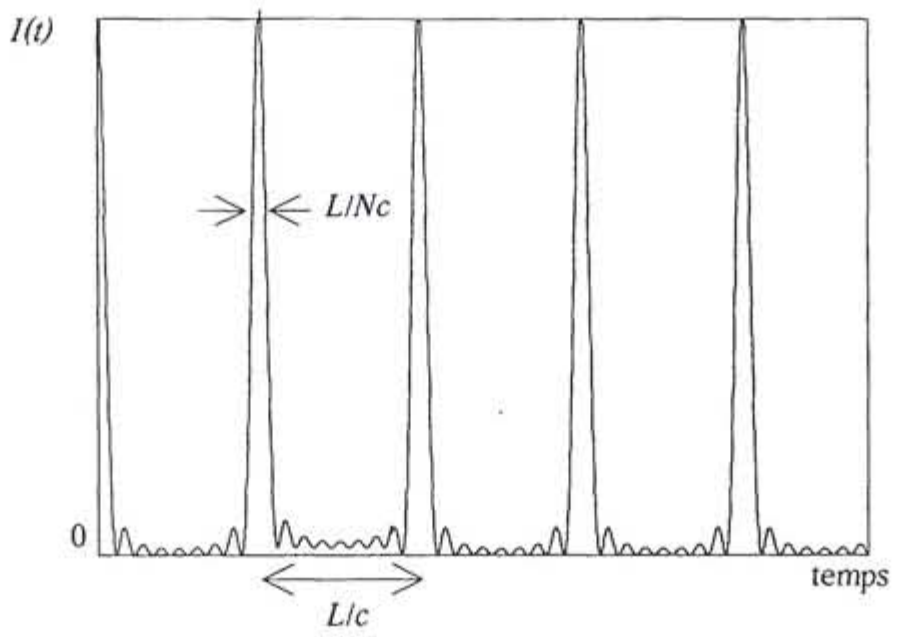

Figure 18

On obtient un train d'impulsions lumineuses dont la période de répétition $T$ vaut

$$
\mathrm{T}=\frac{2 \pi}{\Delta}=\frac{\mathrm{L}}{\mathrm{c}}
$$

c'est-à-dire le temps nécessaire à l'impulsion pour faire un tour dans la cavité. Il n'y a donc pour le champ intracavité qu'une seule impulsion. La valeur maximale de l'intensité vaut :

$$
\mathrm{I}_{\mathrm{Max}}=\frac{\mathrm{N}^{2} \mathrm{a}_{0}^{2}}{2}=\mathrm{NI}
$$

La dépendance en $N^{2}$ montre bien qu'il y a alors interférence totalement constructive entre les $N$ modes lasers. La durée de chaque impulsion vaut alors :

$$
\frac{2 \pi}{N \Delta}=\frac{T}{N}
$$

L'énergie contenue dans chaque impulsion est donc proportionnelle à $N$. Remarquons que la durée de ces impulsions est d'autant plus courte que $N$ est grand. Ce nombre est de l'ordre de $\Delta \omega \mathrm{L} / \mathrm{c}$ où $\Delta \omega$ est la largeur de la courbe de gain. Les lasers à modes synchronisés produisant les impulsions les plus courtes sont donc les lasers qui, en régime continu, sont accordables sur une large gamme : lasers à colorant, laser Titane-Saphir.

Mentionnons une autre méthode pour synchroniser les modes : elle consiste à placer dans la cavité un absorbant saturable, milieu ayant des pertes importantes à faible intensité et dont l'absorption s'annule pratiquement lorsque l'intensité qui le traverse est grande. Si le laser fonctionne en régime multimode incorrélé, l'intensité n'est pas suffisante pour "ouvrir 
l'obturateur ». Seule une impulsion géante, créée par la synchronisation des différents modes, est susceptible de rendre l'absorbant saturable transparent : c'est ce régime qu'adopte le laser en régime permanent.

\subsection{Laser relaxé}

Nous avons vu que des lasers comme les lasers à 3 niveaux étaient susceptibles d'émettre spontanément des impulsions (oscillations de relaxation) en présence d'un pompage constant dans le temps. Selon les valeurs des différentes constantes de temps intervenant dans les équations, on constate que les équations d'évolution n'ont pas de solution stationnaire stable mais peuvent avoir des solutions dont la dépendance temporelle est plus compliquée. Cela peut se produire aussi pour certains types de lasers à 4 niveaux. Ce type de fonctionnement sera étudié plus en détail dans le cours sur les instabilités. Les impulsions ainsi obtenues sont en général beaucoup plus longues que celles produites par un laser à modes synchronisés.

\subsection{Laser déclenché}

Une autre technique utilisée pour produire des impulsions consiste à utiliser un obturateur rapide à l'intérieur de la cavité laser ("Q Switch»), commandé extérieurement. Lorsqu'il est fermé, le pompage permet d'accumuler la population dans le niveau supérieur de la transition laser, selon les équations (85). Lorsqu'il est ouvert, l'énergie ainsi stockée est émise en une seule impulsion, beaucoup plus intense que la valeur stationnaire que l'on obtiendrait avec le même taux de pompage. On peut aussi utiliser, de manière analogue au $\S 7.1$, un absorbant saturable qui «ferme » la cavité pour les champs de faible intensité et ne l'ouvre qu'en régime impulsionnel. Un tel système laser n'a pas de régime continu stable, mais est susceptible d'émettre une impulsion ou un train d'impulsions de grande intensité.

\section{PROPRIÉTÉS DE LA LUMIĖRE LASER}

Pour certaines applications, on n'hésite pas à consacrer trois cent mille francs pour l'achat d'un laser à argon de $15 \mathrm{~W}$ de puissance, alors qu'une lampe à incandescence de $150 \mathrm{~W}$ ne coûte que dix francs. La lumière laser doit donc avoir des propriétés particulièrement intéressantes pour justifier une telle dépense. C'est ce que nous allons voir dans les paragraphes suivants.

\subsection{Source ordinaire de lumière}

Reprenons l'exemple de la lampe à incandescence, assimilable en première approximation à un corps noir à $3000 \mathrm{~K}$, qui émet un rayonnement ayant une luminance d'environ $500 \mathrm{~W} / \mathrm{cm}^{2}$ sur un intervalle de longueur d'onde allant de l'infrarouge au vert $\left(2 \times 10^{14} \mathrm{~Hz}\right.$ environ). Pour utiliser cette lumière il nous faut la concentrer à l'aide de lentilles ou de miroirs. Mais il existe une loi de l'optique qui indique que la luminance se conserve entre l'objet et l'image, ou bien décroît dans le cas d'un système absorbant (si cette quantité pouvait augmenter, on pourrait porter un corps noir placé à l'emplacement de l'image à une température supérieure au corps noir objet et violer le second principe de la thermodynamique). On n'obtiendra donc jamais avec cette lampe mieux que $500 \mathrm{~W} / \mathrm{cm}^{2}$ soit une luminance spectrale de $1 \mu \mathrm{W} / \mathrm{cm}^{2} / \mathrm{MHz}$. Nous allons voir qu'il n'en est pas de même pour la lumière laser, qui peut être extrêmement 
concentrée, soit dans le domaine spectral ou temporel (cohérence temporelle) soit dans l'espace (cohérence spatiale).

\subsection{Cohérence temporelle de la lumière laser}

Si on utilise un laser continu monomode par exemple, la lumière est émise dans un intervalle extrêmement étroit de fréquence. Nous avons vu en effet que la fréquence d'un laser monomode était fixée uniquement par la longueur de la cavité (équation (3)). La largeur spectrale du laser, principalement liée aux fluctuations de la longueur optique de la cavité, peut être de l'ordre du $\mathrm{MHz}$. On gagne donc un facteur $10^{7}$ en densité spectrale par rapport aux sources thermiques, si on utilise des lasers monomodes continus.

D'autre part, nous avons vu qu'il était possible de concentrer la puissance laser dans un faible intervalle de temps, et de produire des impulsions ayant des puissances crête très importantes : la technique de synchronisation de mode exposée dans le paragraphe 7.1 permet de dépasser un éclairement instantané de $10^{15} \mathrm{~W} / \mathrm{cm}^{2}$ au maximum de l'impulsion. Dans ces conditions le champ électrique de l'onde lumineuse est supérieur au champ électrostatique exercé par le proton sur l'électron d'un atome d'hydrogène dans son état fondamental. On accède ainsi à une nouvelle échelle d'énergie dans l'interaction lumière matière.

\subsection{Cohérence spatiale de la lumière laser}

La cohérence spatiale de la lumière laser se manifeste par le fait qu'elle est émise sous la forme d'un mode Gaussien parfaitement défini (qui bien souvent est un mode $\mathrm{TE}_{00}$ ). Il s'agit d'une source dont les propriétés spatiales sont limitées par la diffraction, à la différence de sources classiques qui sont des sources étendues où les différents points émettent des ondes sans relation de phase fixes entre elles. Cette cohérence spatiale lui permet d'être concentrable dans l'espace lorsqu'on la focalise avec une lentille, ou dans l'espace des vecteurs d'onde lorsqu'on produit un faisceau collimaté

La lumière laser permet ainsi de produire une bonne approximation de la droite géométrique, utilisé par exemples dans les lasers d'alignements, puisque le faisceau a une très faible divergence (dont le demi-angle au sommet vaut $\lambda / \pi w_{0}$, où $w_{0}$ est la taille du waist), de l'ordre de $10^{-3}$ à $10^{-4}$, mais qui peut être encore réduite en utilisant des systèmes afocaux de type télescope, qui augmentent la valeur de $w_{0}$. On peut par exemple en utilisant des télescopes d'astronomie, produire des faisceaux Gaussiens dont le waist est de l'ordre de $1 \mathrm{~m}$ donc de divergence inférieure à $10^{-6}$ que l'on utilise pour mesurer la distance terre-lune (la tache laser sur la lune a un diamètre de l'ordre de la centaine de mètres).

$\mathrm{Si}$ on utilise un dispositif de focalisation, on peut obtenir une bonne approximation du point géométrique, utilisé par exemple pour le stockage optique de l'information (disques compacts). Plus précisément, un faisceau Gaussien caractérisé par un waist $w_{0}$ qui traverse une lentille de focale $F$ est transformé en un nouveau faisceau Gaussien dont la taille du waist w' ${ }_{0}$ est donnée par :

$$
w_{0}^{\prime}=w_{0}\left[\left(1-\frac{d}{F}\right)^{2}+\frac{z_{R}^{2}}{F^{2}}\right]^{-1 / 2}
$$

où $d$ est la distance du waist objet $w_{0}$ à la lentille et. $\mathrm{z}_{\mathrm{R}}=\frac{\pi \mathrm{w}_{0}^{2}}{2 \lambda}$. La distance du waist image $w_{0}^{\prime}$ à la lentille étant donnée par : 


$$
d^{\prime}=F \frac{d(d-F)+z_{R}^{2}}{(d-F)^{2}+z_{R}^{2}}
$$

En particulier, si on met à l'emplacement du waist objet $(d=0)$ une lentille de courte focale $\left(\mathrm{F}<<\mathrm{z}_{\mathrm{R}}\right)$ :

$$
\mathrm{w}_{0} \approx \frac{\lambda \mathrm{F}}{\pi \mathrm{w}_{0}} \quad \mathrm{~d}^{\prime} \approx \mathrm{F}
$$

Pour des lentilles de grande ouverture numérique on peut utiliser des faisceaux incidents tels que $w_{0} \approx F / \pi$. On obtient alors une tache focale qui a les dimensions de la longueur d'onde; On peut ici aussi gagner à la focalisation un facteur sur l'éclairement beaucoup plus grand qu'avec une source classique.

Pour des raisons pratiques, le mode réel issu d'un laser n'est pas rigoureusement $\mathrm{TEM}_{00}$, ne serait-ce que parce que les différents éléments optiques qui composent la cavité laser ont des dimensions transverses finies. Caractérisons l'abscisse $x_{0}$ du centre réel et le rayon $\rho$ d'un faisceau lumineux dont la distribution transverse d'intensité est $I(x, y)$ par :

$$
x_{0}=\frac{\int x I(x, y) d x d y}{\int I(x, y) d x d y} \quad \rho^{2}=\frac{\int\left(x-x_{0}\right)^{2} I(x, y) d x d y}{\int I(x, y) d x d y}
$$

(on pourrait définir de la même manière l'ordonnée $y_{0}$ du centre du faisceau et son rayon dans le plan $\mathrm{O}_{\mathrm{y}}$. Nous supposerons ici que le faisceau a la symétrie cylindrique autour de son axe de propagation). On montre alors que dans l'espace libre, et à l'approximation paraxiale, $\rho$ varie le long de la direction de propagation $\mathrm{Oz}$ selon la loi

$$
\rho^{2}=\rho_{0}^{2}+\Theta^{2}\left(z-z_{0}\right)^{2}
$$

$z_{0}$ est la position du col ou waist du faisceau réel, où il a le rayon $\rho_{0}$, et $\Theta$ sa demidivergence en champ lointain. De la même manière que les lois de la "mécanique ondulatoire " imposent la relation de Heisenberg sur les variances de $x$ et $p$, les lois de la diffraction imposent une limite inférieure sur le produit $\rho_{0} \Theta$ :

$$
\rho_{0} \Theta \geq \frac{\lambda}{\pi}
$$

On pose alors :

$$
\mathrm{M}^{2}=\frac{\pi \rho_{0} \Theta}{\lambda}
$$

$M^{2}$ est donc un nombre supérieur à 1 qui caractérise l'écart à la limite de diffraction du faisceau réel. Son intérêt est qu'il est invariant lorsque le faisceau lumineux passe par des systèmes optiques paraxiaux non aberrants et sans diaphragmes (lentilles, miroirs...). Plus précisément, la quantité complexe :

$$
q=z-z_{0}+i \frac{\rho_{0}}{\Theta}=z-z_{0}+i \frac{\pi \rho_{0}^{2}}{M^{2} \lambda}
$$

obéit à la règle « $\mathrm{A}, \mathrm{B}, \mathrm{C}, \mathrm{D}$ » de l'optique matricielle, avec $M$ constant, de la même manière que la quantité correspondante $\mathrm{q}=\mathrm{z}-\mathrm{z}_{0}+\mathrm{i} \pi \mathrm{w}_{0}^{2} / \lambda$ du faisceau théorique TEM $\mathrm{o}_{00}$. Retenons qu'un faisceau laser réel diverge $M^{2}$ fois plus qu'un faisceau TEM $\mathrm{M}_{00}$ de même waist, et que la 
dimension de la tache focale est $M^{2}$ fois plus grande que pour un mode $\mathrm{TEM}_{00}$ pour des conditions de focalisation identiques.

\section{QUELQUES DÉVELOPPEMENTS RÉCENTS}

Si le principe de base des lasers est maintenant bien connu depuis plus de trente ans, la recherche dans ce domaine est cependant loin d'être tarie, tant du point de vue fondamental que d'un point de vue appliqué. J'ai choisi de traiter ici deux exemples de sujets en plein développement, parce qu'ils permettent un nouvel éclairage sur le fonctionnement même du laser, et qu'ils peuvent déboucher sur des applications intéressantes.

\subsection{Le laser sans inversion}

Dans le modèle simple de la partie 2 nous avons vu que le mécanisme de gain optique était lié à la possibilité de créer une inversion de population entre les niveaux $|b\rangle$ et $|a\rangle$, et c'est aussi le cas pour tous les mécanismes de gain laser utilisés dans la réalité jusqu'à présent. Le phénomène de base de l'amplification n'est cependant pas cette inversion, mais plutôt l'émission stimulée. Ce n'est que parce que émission stimulée et absorption sont habituellement inséparables que l'inversion de population est nécessaire : dans ce cas seulement l'émission stimulée l'emporte sur l'absorption.

Il en résulte que si, d'une manière ou d'une autre, on rompt ce lien entre absorption et émission, on pourra alors avoir émission stimulée, donc amplification, dès qu'il existera une population non nulle dans l' état $|b\rangle$, même si cette population n'est pas supérieur à celle de l'ètat $|a\rangle$. Or ce lien n'est pas fondamental. Prenons par exemple le cas de l'interaction entre un atome et un rayonnement de très courte longueur d'onde (rayon $\gamma . .$. ) : le transfert d'impulsion lors de l'interaction matière rayonnement est alors très important, et il est de signe différent lors de l'absorption et lors de l'émission. Les raies d'absorption et d'émission, pour des transitions suffisamment étroites, sont alors séparées et une onde peut être résonnante pour l'émission stimulée sans l'être par l'absorption. Cette situation ne peut malheureusement pas dans l'état actuel des connaissances être utilisée pour réaliser de l'amplification.
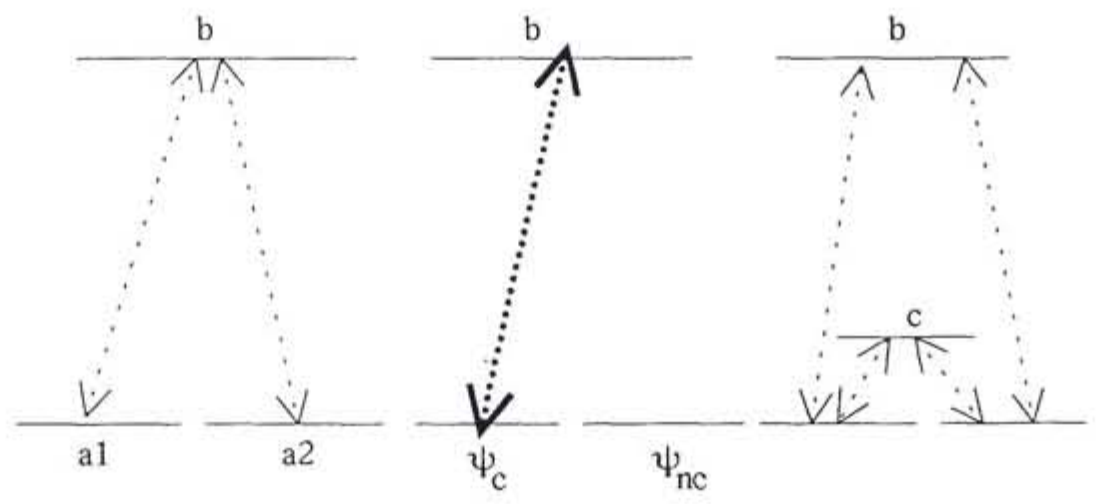

Figure 19 
Une situation plus réaliste existe en particulier dans le cas où le niveau inférieur de la transition est remplacé par 2 niveaux $\left|a_{1}\right\rangle$ et $\left|a_{2}\right\rangle$ (figure 19), supposés dégénérés ou quasi dégénérés. Soient $d_{1}=\left\langle b|\hat{D}| a_{1}\right\rangle$ et $d_{2}=\left\langle b|\hat{D}| a_{2}\right\rangle$ les éléments de matrice (supposés réels) du dipôle électrique sur les 2 transitions optiques $\left|a_{1}\right\rangle \rightarrow|b\rangle$ et $\left|a_{2}\right\rangle \rightarrow|b\rangle$. Considérons alors les deux états quantiques suivants du système, qui forment une nouvelle base de l'espace sous tendu par les vecteurs $\left|a_{1}\right\rangle$ et $\left|a_{2}\right\rangle$ :

$$
\left\{\begin{array}{l}
\left|\psi_{N C}\right\rangle=\frac{1}{\sqrt{d_{1}^{2}+d_{2}^{2}}}\left(d_{2}\left|a_{1}\right\rangle-d_{1}\left|a_{2}\right\rangle\right) \\
\left|\psi_{C}\right\rangle=\frac{1}{\sqrt{d_{1}^{2}+d_{2}^{2}}}\left(d_{2}\left|a_{1}\right\rangle+d_{1}\left|a_{2}\right\rangle\right)
\end{array}\right.
$$

Il est facile de voir que $\left\langle\mathrm{b}|\hat{\mathrm{D}}| \psi_{\mathrm{NC}}\right\rangle=0$ alors que $\left\langle\mathrm{b}|\hat{\mathrm{D}}| \psi_{\mathrm{C}}\right\rangle=\frac{2 \mathrm{~d}_{1} \mathrm{~d}_{2}}{\sqrt{\mathrm{d}_{1}^{2}+\mathrm{d}_{2}^{2}}} \neq 0$. L'état $\left|\psi_{\mathrm{NC}}\right\rangle$ n'est donc pas couplé au rayonnement, même si $\omega=\omega_{0}$. Ce type d'état appelé " état piège », est à l'origine du phénomène de « résonance noire " étudiée depuis de nombreuses années en physique atomique. Le taux de transitions induites par un champ de fréquence $\omega_{0}$ sera dans cette configuration proportionnel à la population du niveau $|b\rangle$ pour ce qui concerne l'émission stimulée responsable du gain laser, et à la population de l'état $\left|\psi_{C}\right\rangle$ pour ce qui concerne l'absorption. Si on arrive à créer dans la multiplicité inférieure un état quantique proche de $\left|\psi_{\mathrm{NC}}\right\rangle$, il y aura amplification, même si la population totale $\mathrm{N}_{\mathrm{a}}$ de la multiplicité inférieure est supérieure à $\mathrm{N}_{\mathrm{b}}$. C'est donc l'interférence destructive entre les deux chemins possibles $\left|\mathrm{a}_{1}\right\rangle \rightarrow$ $|b\rangle$ et $\left|a_{2}\right\rangle \rightarrow|b\rangle$ pour l'absorption qui est à l'origine de la rupture du lien habituel entre émission stimulée et absorption : le laser sans inversion est possible quand une part importante des atomes sont portés dans l'état piégé $\left|\psi_{\mathrm{NC}}\right\rangle$, et donc soustraits du sous-espace des états avec lesquels le niveau supérieur $|b\rangle$ peut avoir des transitions.

Tout le problème est alors de produire de manière efficace cette combinaison très particulière d'état $\left|a_{1}\right\rangle$ et $\left|a_{2}\right\rangle$ qu'est $\left|\psi_{\mathrm{NC}}\right\rangle$, et de nombreuses méthodes ont été proposées pour y arriver. On peut utiliser des trains d'impulsions dont la fréquence de répétition est un multiple de l'écart énergétique entre les niveaux $\left|a_{1}\right\rangle$ et $\left|a_{2}\right\rangle$ ou bien des interférences entre chemins différents d'auto-ionisation d'atomes. Le schéma le plus réaliste consiste à utiliser un autre champ électromagnétique (par exemple RF) qui interagit avec les 2 niveaux $\left|a_{1}\right\rangle$ et $\left|a_{2}\right\rangle$, via un quatrième niveau $|c\rangle$. Un processus de type pompage optique permet alors de peupler l'état $\left|\Psi_{N C}\right\rangle$. Pour obtenir un laser sans inversion, il suffit alors de placer un petit nombre d'atomes dans le niveau supérieur $|b\rangle$, par un processus de pompage annexe, qui n'a pas besoin d'être très efficace. On voit alors tout l'intérêt de ce type d'approche, suggéré en particulier dans la perspective de construire des lasers de courtes longueurs d'onde (VUV ou X) : on peut se placer dans des conditions où une oscillation de type laser est possible en manipulant essentiellement le niveau inférieur de la transition laser (création d'une cohérence entre sousniveaux pour créer l'état $\left|\psi_{\mathrm{NC}}\right\rangle$. En effet pour les transitions atomiques tombant dans le domaine $\mathrm{X}$, le niveau supérieur, de très courte durée de vie, est très difficile à peupler de manière appréciable, et on ne peut réaliser d'inversion de population que par des processus extrêmement violents, mettant en jeu par exemple les lasers utilisés pour la fusion contrôlée 
(voir le cours de P. Jaeglé sur les lasers $X$ ). La création de l'état $\left|\psi_{\mathrm{NC}}\right\rangle$ en utilisant des lasers résonants entre niveaux profonds de l'atome paraît en revanche plus à portée de la main.

De l'idée de base à la réalisation pratique, il y a un long chemin qui est loin d'être parcouru complètement à l'heure actuelle. On enregistre cependant des avancées expérimentales : il a été prouvé expérimentalement qu'on pouvait éliminer l'absorption d'une onde par un atome en manipulant par d'autres champs appliqués le niveau inférieur de la transition («transparence induite électromagnétiquement »). Des résultats préliminaires ont été publiés concernant l'amplification sans inversion, mais aucun laser sans inversion ne fonctionne à l'heure actuelle.

\subsection{Le laser sans seuil}

La formule (65) donnant le taux de pompage minimum nécessaire à l'oscillation laser montre que celui-ci est proportionnel à $\gamma_{\mathrm{b}}$ taux de relaxation du niveau supérieur de la transition laser. Ce niveau étant nécessairement couplé au champ électromagnétique ne serait-ce que sur la transition laser $|\mathrm{b}\rangle \rightarrow|\mathrm{a}\rangle$, la valeur minimale de $\Gamma_{b}$ est le taux de désexcitation spontanée de ce niveau vers tous les niveaux inférieurs. On a longtemps pensé qu'une telle quantité était intrinsèque au système quantique considéré, au même titre que la valeur exacte de ses fréquences de résonance. De récentes études ont montré qu'il n'en était rien, et qu'il était possible de manipuler l'émission spontanée en modifiant l'environnement du système quantique : on peut donc réduire $\gamma_{\mathrm{b}}$ et ainsi réduire la quantité d'énergie minimale pour faire fonctionner un laser. Ici aussi, l'intérêt suscité par ce type d'étude est considérable, notamment en vue d'applications au développement de lasers semi-conducteurs sans seuil (encore appelés diodes électroluminescentes monomodes).

Le taux d'émission spontanée d'un niveau discret $|b\rangle$ d'un système quantique est donné par la règle d'or de Fermi

$$
\Gamma=\sum_{i} \frac{2 \pi}{\hbar} \int\left|\left\langle b\left|\hat{H}_{I}\right| i\right\rangle\right|^{2} \rho_{\varphi}(\Omega) d \Omega
$$

où $H_{l}$ est l'hamiltonien d'interaction entre le champ et l'atome, $|i\rangle$ un niveau atomique d'énergie inférieure au niveau $|b\rangle$ et $\rho_{\varphi}(\Omega)$ la densité d'états finaux accessibles au photon d'émission spontanée de fréquence $\omega_{\mathrm{i}}=\left(\mathrm{E}_{\mathrm{b}}-\mathrm{E}_{\mathrm{i}}\right) / \hbar$ et se propageant dans P'angle solide défini par $\Omega$ à $d \Omega$ près. Cette dernière quantité n'est pas intrinsèque à l'atome considéré mais relative à l'interaction entre celui-ci et son environnement. On montre en particulier qu'elle est fortement modifiée si l'atome est situé dans une cavité résonnante de grande finesse :

- si $\omega_{i}$ est résonnant avec la cavité, la densité augmente : il y a accroissement de l'émission spontanée, ainsi qu'une modification de sa dynamique (car le continuum avec lequel l'atome est couplé a alors une largeur très faible, et on sort du domaine d'application de la règle d'or de Fermi : il peut y avoir en particulier échange oscillant d'énergie entre l'atome et la cavité, et non plus décroissance exponentielle)

- si $\omega_{\mathrm{i}}$ n'est résonnant avec aucune fréquence propre de la cavité, la densité diminue : il y a inhibition de l'émission spontanée, qui est l'effet intéressant pour l'application au laser.

Ce type d'effet est particulièrement marqué dans les cavités résonnantes de type fermé, comme les cavités pour rayonnement micro-onde : en effet la cavité "piège " les photons spontanés quelle que soit la direction $\Omega$ de leur direction d'émission, ou pour des atomes situés entre deux plaques métalliques très proches l'une de l'autre. Des facteurs considérables de modification de l'émission spontanée ont été mesurés dans ces configurations. Mais il est beaucoup plus faible dans les cavités ouvertes de type Fabry-Perot, où les photons d'émission 
spontanée peuvent s'échapper dans le plan transverse à l'axe de la cavité : l'effet premier est dans ce cas une modification du diagramme angulaire de rayonnement spontané, et l'effet global après intégration sur $\Omega$ dépend de l'angle solide des modes oủ les photons sont piégés par rapport à $4 \pi$. Dans le cas de lasers de type "macroscopique », la cavité laser est grande et l'angle solide en question est très faible. Il n'en est pas de même pour les lasers à semiconducteurs, où on peut réaliser des lasers à émission de surface dont la cavité a une longueur de $\lambda$ (pour augmenter l'émission spontanée) ou $\lambda / 2$ (pour la réduire). Des facteurs de réduction de l'émission spontanée de 30 environ ont d'ores et déjà été observés dans de telles configuration.

\section{Bibliographie}

Il existe de nombreux ouvrages généraux sur le fonctionnement des lasers. On pourra par exemple consulter avec profit les ouvrages suivants :

- Grynberg G., Aspect A., Fabre C., «Introduction aux lasers et à l'optique quantique », tomes

1 et 2, Polycopié de l'Ecole Polytechnique (1994),

- Siegman A., "Lasers », University Science Books (1986),

- Sargent M., Scully M., Lamb W., « Laser Physics », Addison-Wesley (1975),

- Yariv A., "Quantum Electronics »,

ainsi que les tomes successifs du "Laser Handbook», T. Arecchi, E. Schultz-Dubois éditeurs, qui présentent des exposés plus détaillés sur différents sujets liés au laser.

Sur les aspects historiques du développement du laser :

-Centennial papers, IEEE Journal of Quantum Electronics, 20 (1984), p. 545,

- Bertolotti M., " Masers and Lasers, a historical approach », Adam-Hilger (1983)

Pour le laser sans inversion, un bon article de revue est

- Mandel P., «Amplification and lasing without inversion», dans «Nonlinear dynamics and spatial complexity in optical systems», The Scottish Universities Summer School in Physics, R. Harrison, J. Uppal editors, SUSSP Publications (1993).

Pour le laser sans seuil, on trouvera une exposition détaillée du sujet dans :

- Haroche S., cours de l'Ecole d'Eté des Houches, paru dans «Systèmes fondamentaux en Optique Quantique», Les Houches, Session 53, J. Dalibard et al. eds, North Holland (1992), pp. 771

- Yamamoto Y., Machida S., Igeta K., Bjork G., dans «Coherence, Amplification and Quantum effects in Semi-Conductor Lasers», Y. Yamamoto ed., Wiley (1991), p. 561, Les ouvrages cités ici contiennent en particulier une liste complète des références des articles originaux, qui n'ont pas été mentionnés dans cette introduction pour des raisons de limitation de place. 